\title{
Remodeling of Conformational Dynamics Enhances Catalytic Activities of M1 Zinc-metallopeptidases from Lanthipeptide Biosynthesis
}

chang zhao

Sichuan University

Wang-Jian Sheng

Nanjing University

ying wang

Nanjing University

Jie Zheng

Nanjing University

Xiangqian Xie

Nanjing University

Yong Liang

Nanjing University https://orcid.org/0000-0001-5026-6710

wanqing wei

Nanjing University

Rui Bao

Department of Laboratory Medicine, State Key Laboratory of Biotherapy and Cancer Center, West China Hospital, Sichuan University and Collaborative Innovation Center

Huan Wang ( $\sim$ wanghuan@nju.edu.cn )

Nanjing University

Article

Keywords: Lanthipeptides, biosynthesis, catalysis

Posted Date: September 14th, 2021

DOI: https://doi.org/10.21203/rs.3.rs-860352/v1

License: (c) (1) This work is licensed under a Creative Commons Attribution 4.0 International License.

Read Full License 
Version of Record: A version of this preprint was published at Nature Chemical Biology on May 5th, 2022. See the published version at https://doi.org/10.1038/s41589-022-01018-2. 

metallopeptidases from Lanthipeptide Biosynthesis

Chang Zhao, ${ }^{1,3}$ Wangjian Sheng,,${ }^{2,3}$ Ying Wang, ${ }^{2}$ Jie Zheng, ${ }^{2}$ Xiangqian Xie, ${ }^{2}$ Yong Liang, ${ }^{2}$ Wanqing Wei, ${ }^{2 *}$ Rui $\mathrm{Bao}^{1 *}$ and Huan Wang $2^{*}$

${ }^{1}$ Center of Infectious Diseases, State Key Laboratory of Biotherapy, West China Hospital, Sichuan University and Collaborative Innovation Center, Chengdu, 610093, China

${ }^{2}$ State Key Laboratory of Coordination Chemistry, Chemistry and Biomedicine Innovation Center of Nanjing University, Jiangsu Key Laboratory of Advanced Organic Materials, School of Chemistry and Chemical Engineering, Nanjing University, Nanjing, 210093, China

${ }^{3}$ These authors contributed equally: Chang Zhao, Wangjian Sheng

*e-mail: wanghuan@nju.edu.cn; baorui@scu.edu.cn; wanqwei@163.com

\section{ABSTRACT}

Lanthipeptides are an important group of natural products with diverse biological functions, and their biosynthesis requires the removal of N-terminal leader peptides (LPs) by designated proteases. LanP $\mathrm{M}_{1}$ enzymes, a subgroup of M1 zincmetallopeptidases, are recently identified as bifunctional proteases with both endo- and aminopeptidase activities to remove LPs of class III and class IV lanthipeptides. Herein, we report the biochemical and structural characterization of EryP as the LanP $\mathrm{M}_{\mathrm{M} 1}$ enzyme from the biosynthesis of class III lanthipeptide erythreapeptin. We determined X-ray crystal structures of EryP in three conformational states, the open, intermediate and closed states and identified a unique interdomain Ca binding site as a regulatory element to modulate its domain dynamics and proteolytic activity. Inspired by the regulatory $\mathrm{Ca}$ binding, we develop a strategy to engineer $\mathrm{LanP}_{\mathrm{M} 1}$ enzymes for enhanced catalytic activities by strengthening inter-domain associations and driving the conformational equilibrium toward their closed forms.

\section{INTRODUCTION}

M1 family zinc-metallopeptidases are widely distributed in many species and regulate a diverse range of biological processes. ${ }^{1}$ For instance, Escherichia coli (E. coli) aminopeptidase $\mathrm{N}(\mathrm{ePepN})$ is involved in ATP-dependent downstream processing during cytosolic protein degradation. ${ }^{2,3}$ Thermoplasma acidophilum tricorn interacting factor F3 (F3) acts downstream of the proteasome to produce di- and tripeptides. ${ }^{4}$ Human endosomal insulin-regulated aminopeptidase (IRAP) is a key regulator in endocytic trafficking and signaling, ${ }^{5}$ while the endoplasmic reticulum aminopeptidases (ERAP1 and ERAP2) are responsible for antigen-trimming. ${ }^{6,7}$ Recently, a new biological function of M1 metallopeptidases is revealed in the biosynthesis of bacteria-derived natural product lanthipeptides, a subfamily of ribosomally synthesized and 
posttranslationally modified peptides (RiPPs). ${ }^{8}$ As the most studied and rapidly growing family of RiPPs, lanthipeptides are featured with diverse structures and biological activities, including antimicrobial, anti-fungal, anti-virus and antiallodynic properties. ${ }^{8}$ The precursor peptide of a lanthipeptide is usually composed of an N-terminal leader peptide and a C-terminal core peptide, which undergoes enzymatic cyclization during biosynthesis. The N-terminal leader peptide plays important roles during the biogenesis of lanthipeptides as recognition elements for modification enzymes, as secretion signals during the exportation or for self-immunity. ${ }^{9}$ The removal of leader peptides is often the last but essential step to produce the mature lanthipeptides. If additional modification is required on the N-terminus of lanthipeptides, such as in the biosynthesis of lipolanthines and goadvionins, ${ }^{10,11}$ precise and efficient removal of the $\mathrm{N}$-terminal leader peptide becomes even more essential.

A subgroup of $\mathrm{M} 1$ zinc-metallopeptidases, named LanP $\mathrm{M}_{1}$, function as key proteases to remove the $\mathrm{N}$-terminal leader peptides (LPs) of class III and class IV lanthipeptides during their maturation. ${ }^{12}$ AplP from the biosynthesis of class III lanthipeptide NAI-112 represents the first example of LanP $\mathrm{M}_{\mathrm{M} 1}$ proteases and removes the leader peptide of precursor peptide AplA after cyclization and glycosylation at its core peptide. ${ }^{13}$ As M1 metallopeptidases usually prefer linear peptides that are shorter than 20 amino acids, ${ }^{14} \mathrm{LanP}_{\mathrm{M} 1}$ enzymes therefore represent a rare subgroup of $\mathrm{M} 1$ metallopeptidases that cleave large cyclic peptide substrates (> 50 amino acid long). ${ }^{12,13,15,16}$ Although the endopeptidase and exopeptidase activities of ePepN remain debatable, ${ }^{2,17,18}$ it is generally observed that M1 metallopeptidases only act on the N-terminus of polypeptides and exhibit no endopeptidase activity. Intriguingly, LanP $\mathrm{P}_{\mathrm{M} 1}$ proteases possess dual enzymatic activities and remove the LP of precursor peptides through a two-stage process: first, LanP $\mathrm{M}_{\mathrm{M} 1}$ cleaves a major N-terminal portion of the $\mathrm{LP}$ as an endopeptidase; second, $\mathrm{LanP}_{\mathrm{M} 1}$ trims off the remaining overhang residues as an aminopeptidase to produce the mature natural products. ${ }^{13}$ The mechanism that LanP $\mathrm{P}_{\mathrm{M} 1}$ enzymes switch between these two functions remains unknown. 


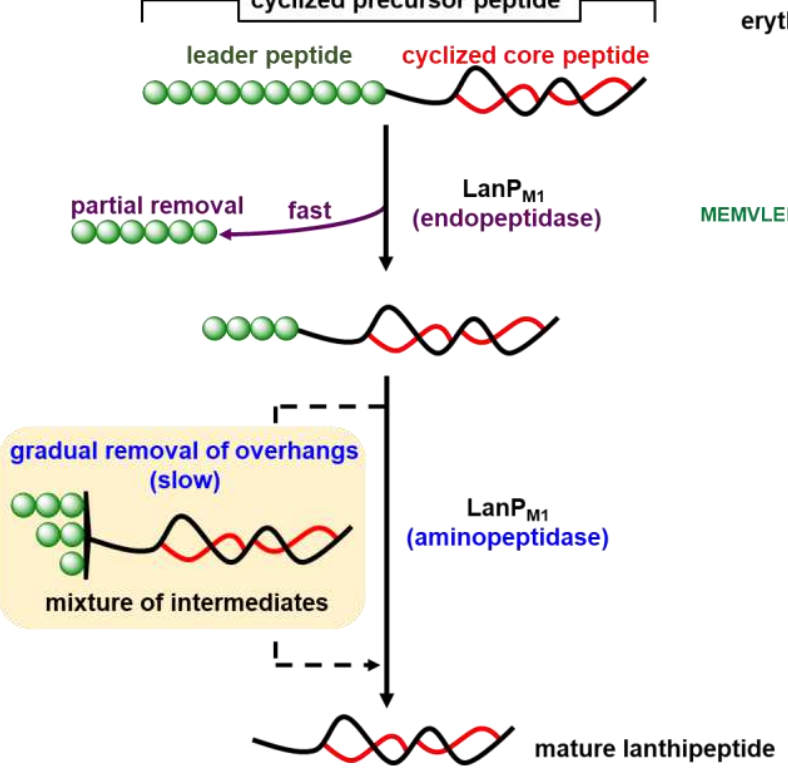
erythreapeptin BGC
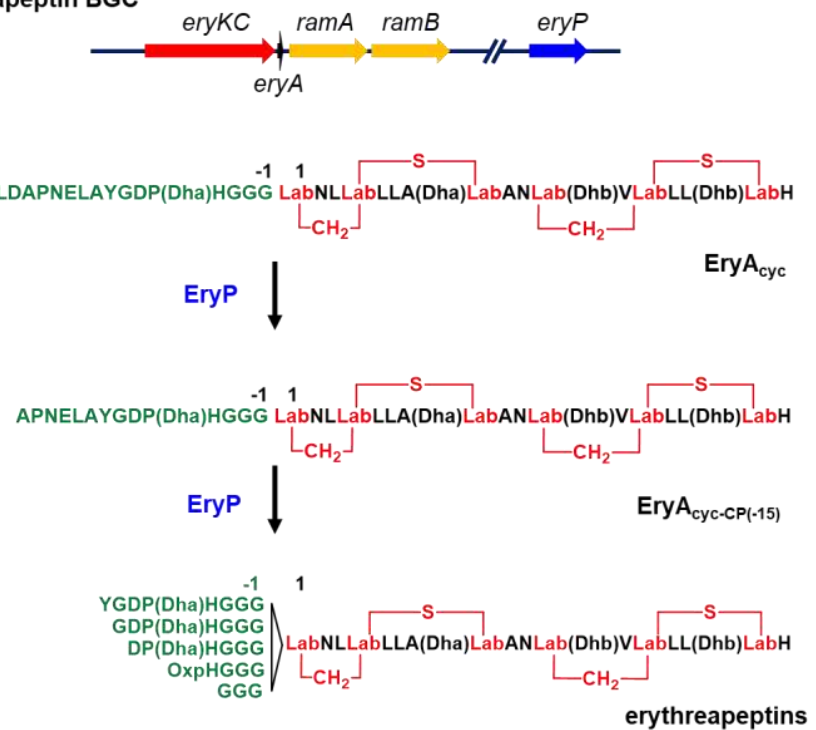

Fig. 1 | LanPM1 enzymes in lanthipeptide biosynthesis. a, Bifunctional LanP $\mathrm{M}_{\mathrm{M} 1}$ enzymes remove LP of lanthipeptides through a two-step process. b. EryP partially removes the LP of cyclized EryA peptide (Ery $\mathrm{A}_{\text {cyc }}$ ) by generating erythreapeptin congeners. The coding gene of EryP is located outside the erythreapeptin BGC.

LanP $\mathrm{P}_{\mathrm{M} 1}$ enzymes are usually highly active as endopeptidases, but significantly less efficient as aminopeptidases during the processing of LP overhang residues. ${ }^{13,16}$ The inefficient aminopeptidase activities of LanP $\mathrm{M}_{\mathrm{M} 1}$ enzymes often result in incomplete LP removal both in vitro and in vivo (Fig. 1a), which impedes the heterologous production and bioengineering of corresponding lanthipeptides. ${ }^{9,}{ }^{12}$ Thus, to understand the enzymology underlining the dual functionality of LanP $\mathrm{P}_{\mathrm{M} 1}$ proteases and to engineer LanP $\mathrm{M}_{\mathrm{M} 1}$ enzymes with improved aminopeptidase activities for lanthipeptide production are highly appealing. Herein, we report the biochemical and structural characterization of EryP, which is the LanP $\mathrm{M}_{\mathrm{M}}$ enzyme from the biosynthesis of class III lanthipeptide erythreapeptin. We determined crystal structures of EryP in three conformational states, the open, intermediate and closed states, by X-ray diffraction and identified a unique inter-domain Ca binding site, which functions as a regulatory element by modulating domain dynamics and the activity of the enzyme. Inspired by the effect of the regulatory $\mathrm{Ca}$ binding, we have developed a practical strategy to acquire engineered LanP $\mathrm{M}_{\mathrm{M} 1}$ mutants with enhanced aminopeptidase activities by strengthening inter-domain associations of LanP $\mathrm{P}_{\mathrm{M} 1}$ enzymes and driving the conformational equilibrium toward their closed forms.

\section{RESULTS}

EryP is a bifunctional LanPM1 protease for leader peptide removal in erythreapeptin biosynthesis. To obtain mechanistic understanding of $\mathrm{LanP}_{\mathrm{M} 1}$ enzymes, we focused on EryP from the biosynthesis of class III lanthipeptide erythreapeptin, which is encoded in the genome of its producing strain Saccharopolyspora erythraea NRRL 2338 but 
located far outside the erythreapeptin BGC (Fig. 1b, Supplementary Fig. 1). ${ }^{13,19}$ EryP was expressed heterologously as a His $_{6}$-tagged fusion protein from E. coli BL21 (DE3) and purified by immobilized metal affinity chromatography as a monomer in solution (Supplementary Fig. 2). His ${ }_{6}$-EryP cleaved EryA leader peptide (EryA $\left.A_{L P}\right)$ by generating fragments $\operatorname{Ery}_{L P}(-15)$ and $\operatorname{Ery}_{L P}(-21)$ as major products, indicating its function as an endopeptidase (Fig. 2a). In addition, His $6^{-}$ EryP exhibited aminopeptidase activity by hydrolyzing amino acid para-nitroanilide ( $p$ NA) derivatives, including Ala$p$ NA, Pro- $p$ NA and Leu- $p$ NA, therefore confirming the dual proteolytic activities of EryP for EryA $A_{L P}$ processing (Fig. $2 b$, Supplementary Fig. 3).

$\operatorname{EryA}_{L P(-21)} \quad \operatorname{EryA}_{L P(-15)}$ MEMVLFELQELD APNELAYGDPSHGGGC

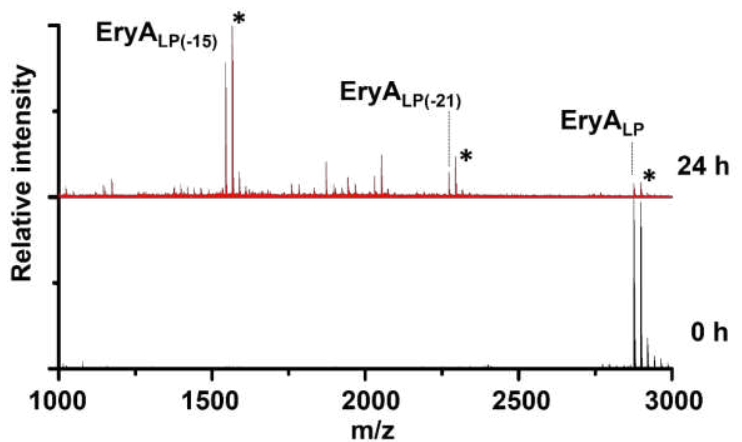

b

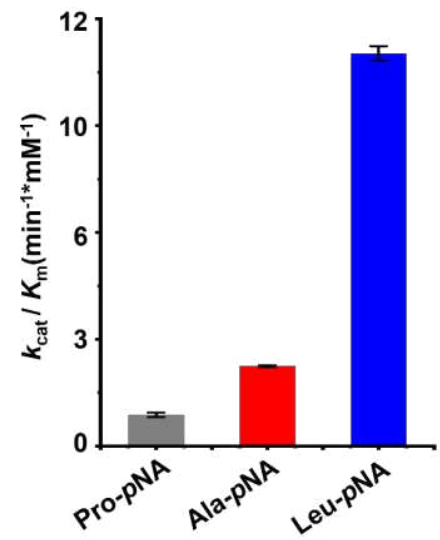

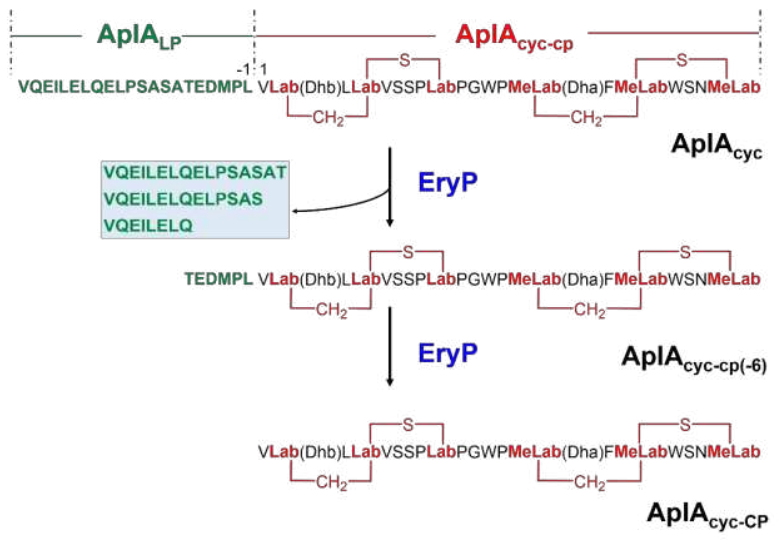

d

ApIA $_{\text {cyc-CP(-6) }}$ ApIA cyc-CP(-3) VQEILELQ-ELPSAS-A $\left.\right|_{-6}$ T-ED $\left.\right|_{-3}$ MPL-(ApIA $A_{\text {cyc-CP }}$ )

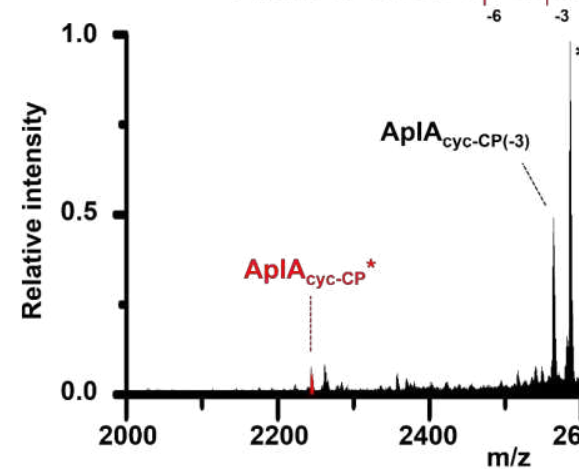

Fig. 2 | Bifunctional protease EryP processes both Ery $A_{L P}$ and Apl $\mathbf{A}_{c y c}$ peptides. a, MS spectrum of EryA $A_{L P}$ peptide cleaved by EryP at multiple sites as an endopeptidase. EryA $\mathrm{LP}_{\mathrm{LP}}: M_{\mathrm{calc}}=2876.18 \mathrm{Da}, M_{\mathrm{obs}}=2876.60 \mathrm{Da}$; $\operatorname{EryA} \mathrm{A}_{\mathrm{LP}}(-15)$ : $M_{\text {calc. }}=1544.62 \mathrm{Da}, M_{\text {obs. }}=1544.76 \mathrm{Da} ; \operatorname{EryA}_{\mathrm{LP}}(-21): M_{\text {calc. }}=2271.98 \mathrm{Da}, M_{\text {obs. }}=2272.13 \mathrm{Da} . \mathbf{b}$, EryP hydrolyzes amino acid$p$ NA derivatives as an aminopeptidase. Error bars indicate standard deviation of three independent replicates. $\mathbf{c}$, Schematic illustration of EryP removing the LP of AplA $\mathrm{A}_{\text {cyc }}$ peptide through a two-stage process. d, EryP completely removed the leader peptide of $\mathrm{AplA}_{\mathrm{cyc}}$ by generating AplA $\mathrm{cyc- \textrm {CP }}$ as the final product. AplA $\mathrm{Acyc}_{\mathrm{CP}}: M_{\mathrm{calc}}{ }^{*}=2242.95 \mathrm{Da}, M_{\mathrm{obs}}{ }^{*}=2243.42 \mathrm{Da}$; $\mathrm{AplA}_{\text {cyc-CP(-3) }}: M_{\text {calc. }}{ }^{*}=2584.12 \mathrm{Da}, M_{\text {obs. }}{ }^{*}=2584.76 \mathrm{Da} ; \mathrm{AplA}_{\text {cyc-CP(-6): }}: M_{\text {calc. }}{ }^{*}=2929.24 \mathrm{Da}, M_{\text {obs. }}{ }^{*}=2928.94 \mathrm{Da}$. Labels ${ }^{*}$ and \# represent sodium and potassium adducts of corresponding peptides, respectively. 
Next, we examined the proteolytic activity of EryP toward cyclized lanthipeptide substrates. Since cyclized EryA peptide $\left(\right.$ EryA $_{\text {cyc }}$, Fig. 1b), the native substrate of EryP, was not accessible, we employed cyclized AplA, the precursor peptide of NAI-112, as an alternative substrate. Cyclized AplA ( $\left.\mathrm{AplA}_{\mathrm{cyc}}\right)$ shares structural similarity with EryA $\mathrm{A}_{\mathrm{cyc}}$ in the leader peptide and the core peptide, therefore serving as a good substrate mimic (Fig. 2c, Supplementary Fig. 4). As expected, EryP cleaved AplA $A_{c y c}$ at multiple sites in the segment of $A p l A_{L P}$ peptide after 90 min incubation by generating $\mathrm{AplA}_{\mathrm{cyc}-\mathrm{CP}(-6)}$ as the detectable core peptide product (Supplementary Fig. 5). Prolonged incubation led to stepwise removal of overhang residues in $\mathrm{AplA}_{\mathrm{cyc}-\mathrm{CP}(-6)}$ and eventually yielded $\mathrm{AplP}_{\mathrm{cyc}-\mathrm{CP}}$ as the final product (Fig. 2d). These results indicated that EryP possesses relaxed substrate specificity and is capable of completely removing LPs of class III lanthipeptides other than its native substrate. It is noteworthy that as an endopeptidase, EryP exhibited distinct preference of cleavage sites in Ery $_{L P}$ and AplP $_{L P}$ : it cleaved EryA $A_{L P}$ at the N-terminus of ELQ and APN motifs but preferred the C-terminus of ELQ and S-A-(S/T) motifs towards AplA $\mathrm{LP}_{\mathrm{LP}}$, indicating that the site-selectivity of EryP for endoproteolytic cleavage might be substrate-dependent (Fig. 2a and Supplementary Fig. 5).

\begin{tabular}{|c|c|c|c|}
\hline \multirow{2}{*}{ Peptides } & \multicolumn{3}{|c|}{$\boldsymbol{K}_{\mathrm{D}} / \boldsymbol{\mu M}$} \\
\cline { 2 - 4 } & EryP & EryP $_{\text {E802R }}$ & ApIP \\
\hline EryA $_{\text {LP }}$ & $40 \pm 6$ & $32 \pm 14$ & N.D. \\
\hline ApIA $_{\text {cyc }}$ & $0.50 \pm 0.11$ & $0.57 \pm 0.08$ & $0.36 \pm 0.18$ \\
\hline ApIA $_{\text {LP }}$ & $8.0 \pm 2.7$ & $4.0 \pm 0.6$ & $2.3 \pm 0.5$ \\
\hline ApIA $_{\text {cyc-CP }}$ & $720 \pm 18$ & N.D. & N.D. \\
\hline
\end{tabular}

Table 1 | Binding affinities of EryP, EryP E802R and ApIP with peptide substrates. N.D. = not determined

To understand the substrate recognition mechanism of EryP, we performed microscale thermophoresis (MST) to determine the binding affinity between EryP and peptide substrates. Results showed that EryP binds to Ery $\mathrm{A}_{\mathrm{LP}}$ with a $K_{D}$ of $(40.8 \pm 5.8) \mu \mathrm{M}$, whereas its binding with $\operatorname{EryA}_{\mathrm{LP}(-15)}$ is too weak to measure (Table 1). EryP binds to full length $\mathrm{Apl}_{\mathrm{cyc}}$ and AplA $\mathrm{L}_{\mathrm{LP}}$ with $K_{D}$ values of $(0.503 \pm 0.109) \mu \mathrm{M}$ and $(7.98 \pm 2.72) \mu \mathrm{M}$, respectively, indicating that the cyclized AplA core

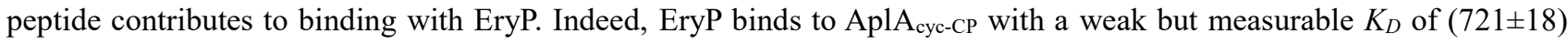
$\mu \mathrm{M}$. Similarly, AplP binds to full length AplA $\mathrm{A}_{\mathrm{cyc}}$ with a $K_{D} \sim 9$-fold lower than that of AplA $\mathrm{LP}_{\mathrm{LP}}$ (Table 1). Therefore, the substrate recognition of EryP and AplP primarily depends on their binding with leader peptides, whereas core peptides act as complementary binding elements.

Overall structure and active-site architecture of EryP. Three sets of EryP crystal structures were determined in distinct conformations, which are postulated as closed, intermediate and open states, with resolution limits from 1.80-2.66 Å (Fig. 3a). The structure of the closed conformation was solved by single isomorphous replacement with anomalous scattering from a Sederivative (SAD), and was used as a model to solve the other two structures by molecular replacement. Similar 
to tricorn-interacting factor F3 (TIFF3) and ePepN of the M1 metalloprotease family, EryP folds into four distinct domains: domain-I (residues 1-201), domain-II (residues 202-456), domain-III (residues 457-552) and domain-IV (residues 553860) (Fig. 3a). The barrel-like N-terminal domain-I is packed by a central 12 -stranded $\beta$-sheet and two smaller antiparallel

$117 \beta$-sheets. The following catalytic domain-II adopts a thermolysin-like fold characterized by an $\alpha / \beta$ lobe on the top of an $\alpha$ helices lobe. A $\mathrm{Zn}$ ion binding motif, $\operatorname{HEXXH}(\mathrm{X})_{18} \mathrm{E}$, which is highly conserved among M1 metallopeptidases, is located at the deep groove between the two lobes (Supplementary Fig. 6). Domain-III presents a barrel-like structure formed by eight $\beta$-strands. The $\mathrm{C}$-terminal domain-IV consists of $16 \alpha$-helices that are organized in an antiparallel manner by forming a bowl-shaped structure, which might regulate the accessibility of the catalytic pocket in domain-II. ${ }^{6}$

In the closed state of EryP, a zinc atom resides at the bottom of active-site cleft in catalytic domain and is coordinated by NE2 atoms of two histidine residues (H306 and H310) and OD atoms of E329 (Fig. 3b, Supplementary Fig. 6) ${ }^{20}$ A catalytic water molecule lies immediately $(2.0 \AA)$ next to the $\mathrm{Zn}$ ion, resulting in a distorted trigonal-pyramidal coordination network (Fig. 3b). The presence of a catalytic water molecule in the active site prior to peptide substrate binding is consistent with previous studies of M1 metallopeptidases, such as thermolysin, and is considered to be essential for their enzymatic functions. ${ }^{20}$ Residue E307 locates $3.3 \AA$ from the water molecule and might participate in catalytic solvent activation as a general base. The highly conserved Y392 resides in the catalytic pocket and might participate in the proteolysis by stabilizing the tetrahedral intermediate formed by water addition to the substrate scissile amide bond. ${ }^{21}$ Both endopeptidase and aminopeptidase activities of EryP $\mathrm{E}_{\mathrm{E} 307 \mathrm{Q}}$ and EryP $\mathrm{P}_{\mathrm{Y} 392 \mathrm{~F}}$ were abolished, confirming the essential role of these residues for catalysis (Supplementary Fig. 7).

The interactions between short peptide substrates and the reaction center were visualized by docking a dipeptide LeuGlu into the active site of EryP in the closed state (Supplementary Fig. 8). The highly conserved $\mathrm{G}_{272} \mathrm{AME}_{275} \mathrm{~N}$ motif in EryP binds to the dipeptide substrate through multiple hydrogen bonds in the docking model. In particular, residue E275 directly binds to the amino group at the N-terminus of the dipeptide with additional binding from residues E132 and E329 (Supplementary Fig. 8). Furthermore, this docking model suggests that both S1 and S1' pockets are spacious to accommodate amino acid side chains of various sizes in peptide substrates, allowing the enzyme to sequentially cleave various residues from the leader of EryA (Supplementary Fig. 8). 
a

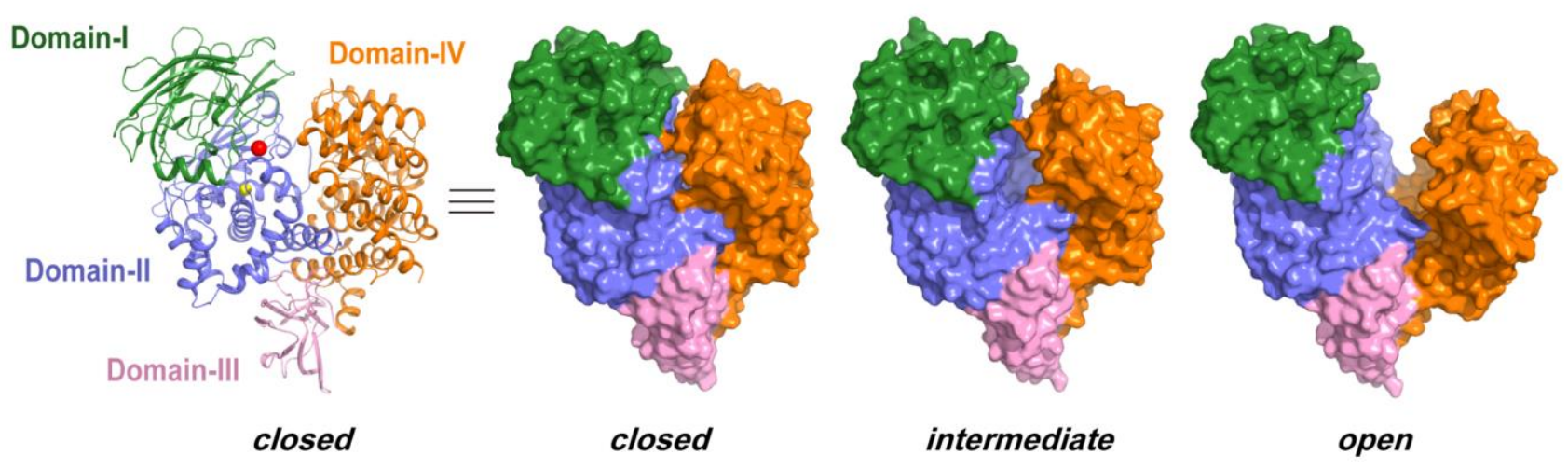

b

C

d
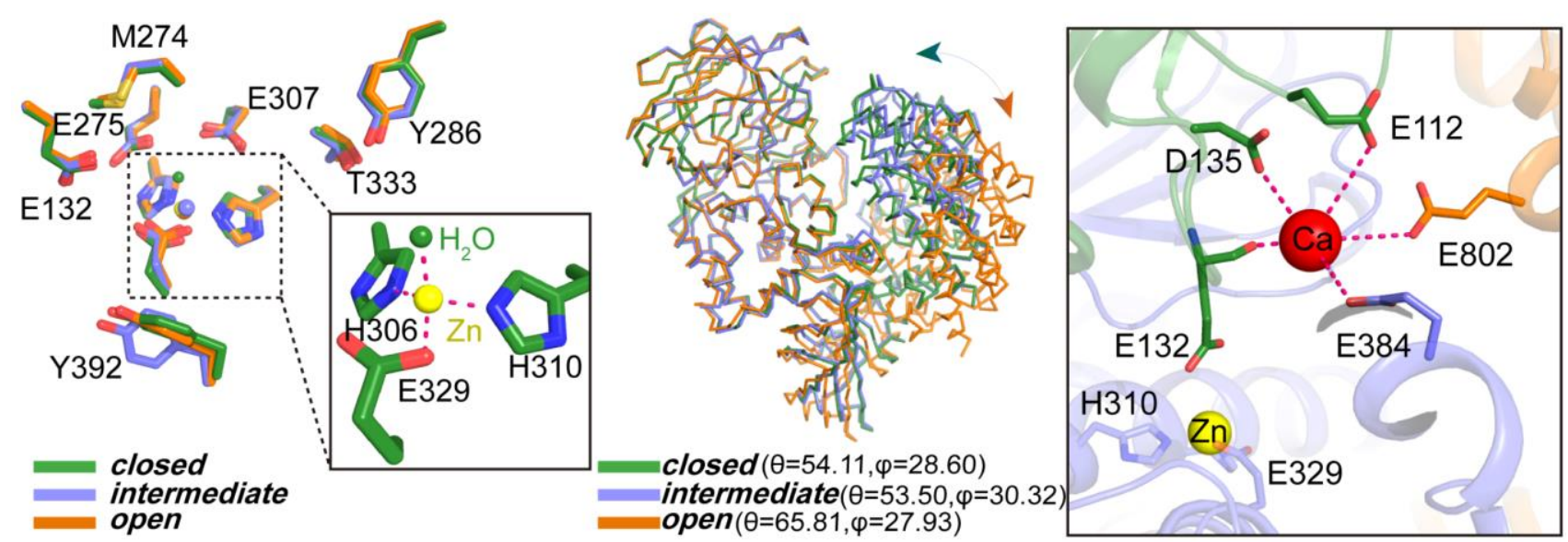

Fig. 3 | Overall structure and the metal ion binding sites of EryP. a, Three sets of crystal structures of EryP. The four domains of EryP are shown in forest green (domain-I), slate (catalytic domain-II), pink (domain-III), and orange (domainIV). The catalytic $\mathrm{Zn}$ atom is shown as a yellow sphere, and the $\mathrm{Ca}$ atom is shown as a red sphere in the closed state. $\mathbf{b}$, Superposition of the active site of EryP in closed (forest green), intermediate (slate) and open (orange) states. The $\mathrm{Zn}$ atom and the catalytic water molecule are shown in yellow and forest green, respectively, in the closed state. c, Superposition of closed, intermediate and open states of EryP in ribbon style. The interdomain angle $\theta$ is defined as the angle of the centers of mass of domain-I and domain-II, domain-III, and domain-IV. The torsion angle $\varphi$ is measured between the center of mass of domain-I and domain-II, the residues 513 and 551 in domain-III, and the center of mass of domain-IV.2 ${ }^{22}$ The angles were measure in UCSF chimera. ${ }^{23} \mathbf{d}$, The inter-domain Ca ion binding site in the closed state of EryP.

Comparative analysis of various conformations of EryP. Conformational flexibility is a common feature among fourdomain M1 metallopeptidases, including ePepN and ERAP1. ${ }^{24-26}$ Among the three sets of EryP crystal structures, domainI and domain-II are well aligned and share an interface of 36.0-44.3 $\AA^{2}$ in area (RMSD from 0.155-0.313 $\AA$ ) (Fig. 3c). 
II. Scissor motions of domain-IV ( 0.76 to 1.72 degrees) around domain-III ( 0.61 to 11.70 degrees of rotation) were observed in the intermediate and open states (Fig. 3c). In comparison with the closed conformation, the areas of domain interface between domain-II with domain-III and domain-IV are reduced from 878.7 to $851.8-876.7 \AA^{2}$ and from 1334.8 to $1129.6-476.2 \AA^{2}$, respectively, in intermediate and open states. The EryP structure in the open state has an approximate $6852 \AA^{3}$ internal cavity volume between domain-II and domain-IV as calculated by CASTp $3.0,{ }^{27}$ which is highly accessible to solvent. This cavity of EryP is significantly larger than known M1 metallopeptidases in their open conformations. For example, ERAP1 accepts long linear peptide substrates and has a cavity of $5080 \AA^{3}$ between its domainII and IV in its open conformation. ${ }^{14}$ The exceptionally large internal cavity of EryP may facilitate the recruitment of large cyclized peptide substrate during catalysis, as $\mathrm{AplA}_{\text {cyc-CP }}$ alone has an estimated volume of $\sim 2300 \AA^{3}$. In the closed conformation, the volume of this cavity significantly shrinks to $1531 \AA^{3}$, and the cavity is fully encompassed by the domain rims in the closed state. Despite the significant conformation alteration among three EryP structural sets, critical activesite residues did not display significant structural differences (Fig. 3b). Specifically, residues Y392 in the closed and open states of EryP are in almost identical position. This is in contrast with several four-domain M1 metallopeptidases, such as ERAP1, in which the catalytic tyrosine residue orients closer toward the $\mathrm{Zn}$ center to participate amide hydrolysis in its closed conformation than that in its open conformation. ${ }^{14}$

Calcium ion enhances inter-domain interactions and the aminopeptidase activity of EryP. In the closed state of EryP, a calcium ion is located about $12.1 \AA$ from the $\mathrm{Zn}$ ion through coordination by residues E112, E132 and D135 from domain-I, residues E384 from domain-II and E802 from domain-IV (Fig. 3d). Such a Ca ion is missing in the open and intermediate states, which is in accordance with the shift of residue E802 away from E112 and D135 (Supplementary Fig. 9). In addition, the side chain of E384 orients in an opposite direction in the open state in comparison with that in the closed state, and the loop of residues 384-390 could not be modeled in the intermediate conformation likely due to its high flexibility (Supplementary Fig. 9). Together, these data suggest that the Ca ion binding site might function as a regulatory element that mediates the domain dynamics, particularly relative positions of domain-IV to domain-I and II.

To understand the role of $\mathrm{Ca}$ ion in mediating the tri-domain interaction, we performed $200 \mathrm{~ns}$ classical molecular dynamics (MD) simulations based on the crystal structure of EryP in the closed state with or without $\mathrm{Ca}^{2+}$ ion bound. Twodimensional descriptors were selected to describe the motion of domain-IV relative to domain-I and II: d1 is the distance between the CA atoms of E802 and E112, and $\mathbf{d} 2$ is the distance between the CA atoms of E802 and E384 (Supplementary Fig. 10). Considering that $\mathbf{d} \mathbf{1}$ and $\mathbf{d} \mathbf{2}$ in the closed state of EryP is $10.95 \AA$ and $9.22 \AA$, respectively, snapshots are marked as closed conformations if $\mathbf{d} \mathbf{1} \leq 10.95 \AA$ and $\mathbf{d} \mathbf{2} \leq 9.22 \AA$; otherwise, conformational transformation is suggested to occur from closed states to an intermediate or open state. Results indicate that with $\mathrm{Ca}^{2+}$ ion bound, EryP tends to adopt closed conformations with domain-IV in close distance with domain-I and II (Fig. 4a). When the $\mathrm{Ca}^{2+}$ ion was removed from 
EryP, a significantly wider distribution of conformations was observed, a portion of which deviated from the closed conformation (Fig. 4a). When Leu- $p$ NA, the favorite aminopeptidase substrate of EryP, was docked into the active site to mimic the substrate-bound EryP, the binding of $\mathrm{Ca}$ ion showed an even stronger effect on locking EryP in the closed conformations (Fig. 4b). Collectively, these data suggest that by bridging residues from domain-I, II and IV, the Ca ion shifts the conformational equilibrium of EryP toward the closed conformation.

Next, we evaluated the impact of $\mathrm{Ca}$ ions on the proteolytic activity of EryP. Elevation of $\mathrm{Ca}^{2+}$ concentration from 0 to $1.0 \mathrm{mM}$ in assay buffer increased catalytic efficiency of EryP toward amino acid-pNA derivatives by 3-5 folds (Fig. $4 \mathrm{c}$, Supplementary Fig. 3). In addition, both endopeptidase and aminopeptidase activities of EryP toward peptide substrates Ery $A_{L P}$ and $A p l A_{c y c-C P(-4)}$, respectively, were significantly improved by the addition of $\mathrm{CaCl}_{2}$ in reaction buffers (Fig. $4 \mathrm{~d}$ and 4e), although the binding affinity was not altered (Supplementary Fig. 11). To confirm that the response of EryP activity to $\mathrm{Ca}$ ions is resulted from the interdomain $\mathrm{Ca}^{2+}$ binding site, $\mathrm{E} 384$ was mutated into an Ala residue. EryP $\mathrm{P}_{\mathrm{E} 384 \mathrm{~A}}$ mutant retains the same level of aminopeptidase activity toward Ala- $p$ NA as EryP, however, was no longer responsive to the change of $\mathrm{Ca}^{2+}$ concentration (Supplementary Fig. 12). Collectively, our results demonstrate that although not strictly required for activity, the interdomain $\mathrm{Ca}^{2+}$ binding site provides a level adjusting mechanism to stabilize the closed conformation of EryP, which consequently enhances its aminopeptidase activity. 
a

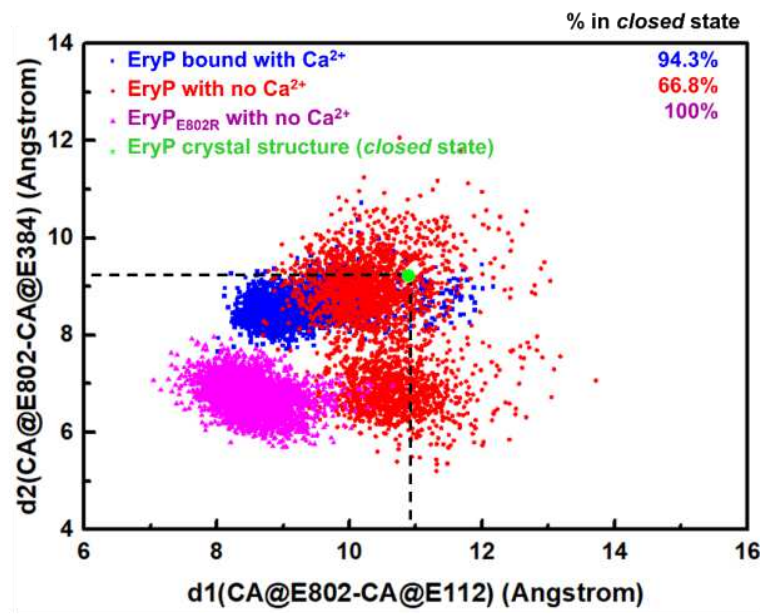

b

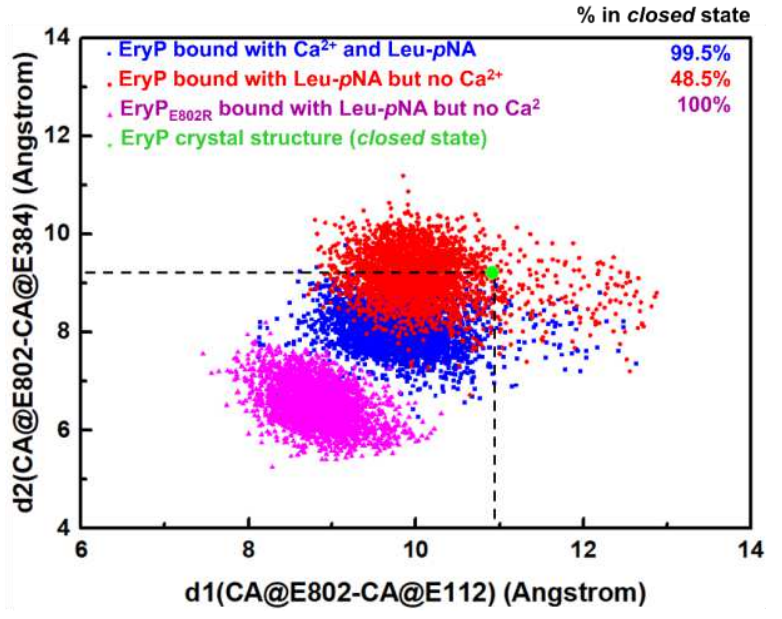

C

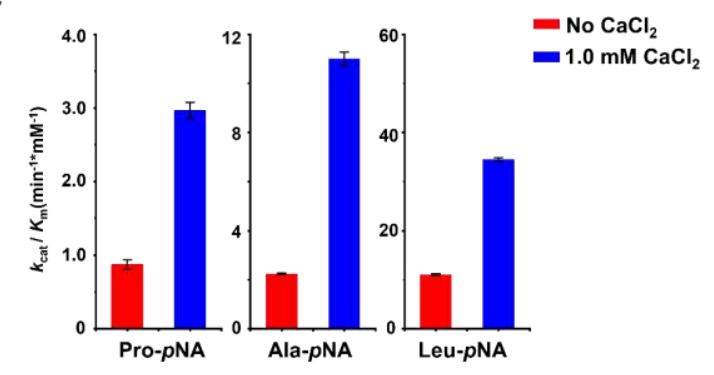

d
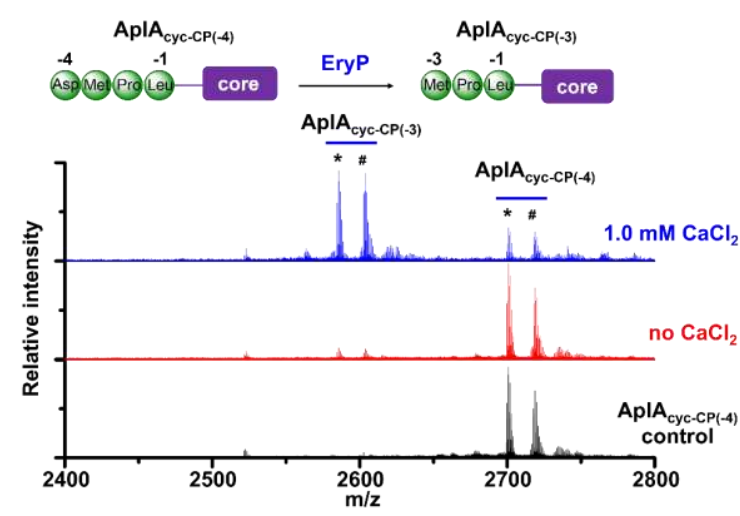

$\operatorname{EryA}_{L P}(-21) \operatorname{EryA}_{L P}(-15)$ MEMVLFELQELDFAPNELAYGDPSHGGG̈C

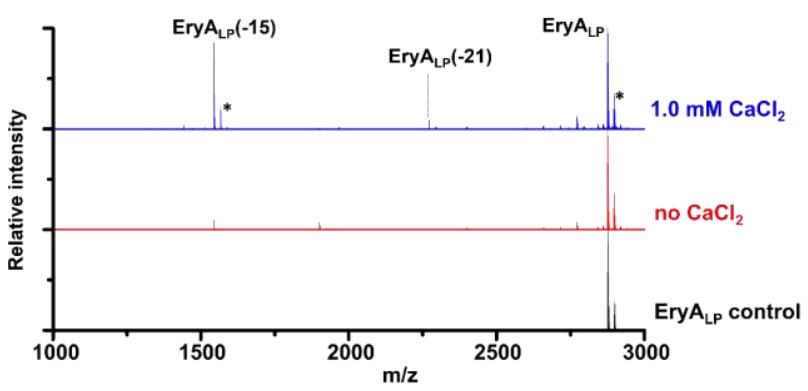

Fig. 4 | The Ca ion binding site regulates the conformational equilibrium and catalytic activity of EryP. a, Distances

$\mathbf{d} \mathbf{1}$ and $\mathbf{d} 2$ in EryP and EryP $\mathrm{E}_{802 \mathrm{R}}$ with or without $\mathrm{Ca}$ ions. b, Distances d1 and d2 in EryP-(Leu- $\left.p \mathrm{NA}\right)$ and EryP $\mathrm{P}_{\mathrm{E} 802 \mathrm{R}}-(\mathrm{Leu}-$ pNA) complexes with or without Ca ions. Data were collected from 20000 snapshots during 200 ns MD simulations. c,

Relative aminopeptidase activity of EryP in the presence or absence of $\mathrm{Ca}$ ions toward three amino acid- $p$ NA derivatives.

Error bars indicate standard deviation of three independent replicates. d, EryP displays enhanced aminopeptidase activity toward peptide substrate $\mathrm{AplA}_{\mathrm{cyc}-\mathrm{CP}(-4)}$ in the presence of $\mathrm{CaCl}_{2} \cdot \mathrm{AplA}_{\mathrm{cyc}-\mathrm{CP}(-3)}: M_{\text {calc. }}{ }^{*}=2584.12 \mathrm{Da}, M_{\mathrm{obs} .}{ }^{*}=2584.77 \mathrm{Da}$; $\mathrm{AplA}_{\text {cyc-CP(-4) }}: M_{\text {calc. }}{ }^{*}=2699.15 \mathrm{Da}, M_{\text {obs. }}{ }^{*}=2700.05 \mathrm{Da} .{ }^{*}$ and ${ }^{\#}$ represents the sodium and potassium adducts of peptides in MS, respectively. e, EryP displays enhanced endopeptidase activity toward Ery $A_{L P}$ in the presence of $\mathrm{CaCl}_{2} . \mathrm{EryA}_{\mathrm{LP}}$ : $M_{\text {calc. }}=2876.18 \mathrm{Da}, M_{\text {obs. }}=2876.63 \mathrm{Da} ; \operatorname{EryA}_{\mathrm{LP}}(-15): M_{\text {calc. }}=1544.62 \mathrm{Da}, M_{\text {obs. }}=1544.27 \mathrm{Da} ; \operatorname{EryA} \mathrm{AP}_{\mathrm{LP}}(-21): M_{\text {calc. }}=2271.98 \mathrm{Da}$, $M_{\text {obs. }}=2271.45 \mathrm{Da}$.

Rational engineering of inter-domain interactions enhances aminopeptidase activities of EryP. The removal of LP overhang residues is the rate-limiting and often incomplete step during the biosynthesis of class III lanthipeptides, and 
therefore LanP $\mathrm{P}_{\mathrm{M} 1}$ proteases with enhanced aminopeptidase activities are highly desired. ${ }^{12,15,16}$ The correlation between structural dynamics and enzymatic activities of EryP provides an opportunity to create an engineered EryP mutant with improved aminopeptidase activity by stabilizing the tri-domain association. The Ca binding residues in EryP provide a promising engineering site to incorporate mutations for enhanced inter-domain network. Based on the closed structure of EryP, we replaced the $\mathrm{Ca}^{2+}$ coordinating residue E802 from domain-IV with an arginine (R) residue, which potentially forms charge-charge interactions with residues E112 and E384 to strengthen the association between domain-I, II and IV. To confirm the engineering effect, we determined the crystal structure of EryP $\mathrm{E}_{\mathrm{E} 82 \mathrm{R}}$ in $1.77 \AA$ resolution (Supplementary Fig. 13). In line with our expectation, the overall structure of EryP $\mathrm{E}_{\mathrm{E} 802 \mathrm{R}}$ displays a more compact conformation than EryP (inter-domain angle $\theta=48.18$, and the torsion angle $\varphi=27.77$ ) mainly due to the newly formed interactions introduced by the E802R mutation (Fig. 5a). Specifically, residue R802 inserts into the cleft of domain II and forms a salt-bridge with residue E384, as well as cation- $\pi$ interaction with residue F387 from domain II (Fig. 5b; Supplementary Fig. 13). Additional interaction between R802 and T133 from domain I was observed, which pulls domain I closer to domain IV and increases their domain interface from 58.2 to $352.3 \AA^{2}$ (Fig. 5a). MD simulation based on the structure of EryP $\mathrm{P}_{\mathrm{E} 802 \mathrm{R}}$ showed that domain-IV of EryP $\mathrm{E} 802 \mathrm{R}_{\mathrm{R}}$ is in significantly closer contact with domain-I and II than that of EryP, and conformations of EryP $_{\mathrm{E} 802 \mathrm{R}}$ are largely maintained in the closed state (Fig. 4a, b). Together, these data support that the E802R mutation strengthens the interactions between domain IV and domain I \& II and drives the conformational distribution of Ery $\mathrm{P}_{\mathrm{E} 802 \mathrm{R}}$ toward a compacted closed state.

Despite the conformational changes altered by the E802R mutation, the majority of active site residues in the catalytic pocket of EryP $\mathrm{E}_{\mathrm{E} 82 \mathrm{R}}$ reside almost identically as in EryP with the exception of Y392, which displays a significant shift with the phenolic oxygen atom approaching the $\mathrm{Zn}$ ion center, shortening their distance from 8.6 to $4.1 \AA$ (Fig. $5 \mathrm{c}$ ). Y392 is proposed to stabilize the tetrahedral intermediate during enzymatic amide hydrolysis (Supplementary Fig. 14), and a closer distance between the $\mathrm{Zn}$ ion and Y392 might facilitate the hydrolytic reaction. ${ }^{21,26,}{ }^{28}$ We next examined whether the proteolytic activity of EryP $\mathrm{E}_{\mathrm{E} 82 \mathrm{R}}$ was improved by the conformational remodeling. By Michaelis-Menten analysis, EryP $_{\mathrm{E} 802 \mathrm{R}}$ exhibited aminopeptidase activity toward amino acid-pNA substrates with $k_{\text {cat }} / K_{\mathrm{m}}$ values increased by $\sim 800$-fold and $K_{\mathrm{m}}$ values lowered by 7-60 folds, indicating that both substrate binding and catalytic efficiency are improved (Fig. $5 \mathrm{~d}$; Supplementary Fig. 15). Meanwhile, the catalytic activity of EryP $\mathrm{E}_{\mathrm{E} 802 \mathrm{R}}$ is no longer responsive to the alteration of Ca ions in the reaction buffer, supporting the notion that the E802R mutation is a reasonable design which could replace the enhancement effect induced by Ca binding (Supplementary Fig. 15). Importantly, EryP $\mathrm{E}_{\mathrm{E} 802 \mathrm{R}}$ showed significantly higher aminopeptidase activity towards peptide $\mathrm{AplA}_{\mathrm{cyc}-\mathrm{CP}(-4)}$ than EryP during the removal of the N-terminal Asp residue (Fig. 5e). Together, our data showed that the E802R mutation stabilizes the overall structure of EryP $\mathrm{E} 802 \mathrm{R}_{\text {in a more compact }}$ closed conformation than EryP, which leads to improved aminopeptidase activity. 
To further validate the origin of the enhanced aminopeptidase activity of Ery $\mathrm{P}_{\mathrm{E} 802 \mathrm{R}}$, we performed MD analysis of the substrate binding of EryP and EryP $\mathrm{E} 802 \mathrm{R}$ to Leu- $p \mathrm{NA}$ (Supplementary Fig. 16). Typically, three major factors govern the course of amide bond hydrolysis: the activation of an amide bond by $\mathrm{Zn}$ ion, the nucleophilic attack by the hydrolytic water and the stabilization of the resulting tetrahedron intermediate. ${ }^{29} \mathrm{MD}$ analysis indicated that the average distance between the $\mathrm{Zn}$ ion and the amide oxygen of Leu- $p \mathrm{NA}$ bound in EryP $\mathrm{E}_{\mathrm{E} 802 \mathrm{R}}$ was $\sim 1.5 \AA$ shorter than that in EryP, suggesting a stronger amide activation and stabilization of the tetrahedron intermediate (Supplementary Fig. 16). In addition, the hydrolytic water is $\sim 0.5 \AA$ closer to the amide $\alpha$-carbon, which increases the likelihood of nucleophilic attack to occur. Finally, the phenolic oxygen of Y392 displays an average distance of $2.0 \AA$ from the amide oxygen, which is $\sim 2.0 \AA$ shorter than that in EryP, leading to a significantly stronger stabilization effect of the tetrahedron intermediate. These results show that the active site constellation of EryP $\mathrm{E}_{\mathrm{E} 02 \mathrm{R}}$ is more optimized for amide hydrolysis than that of EryP.

a

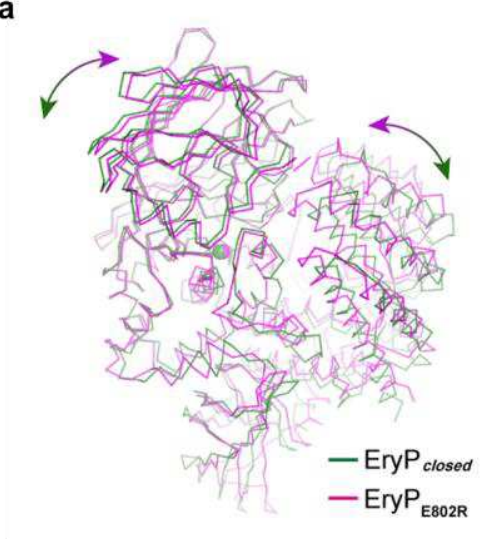

b

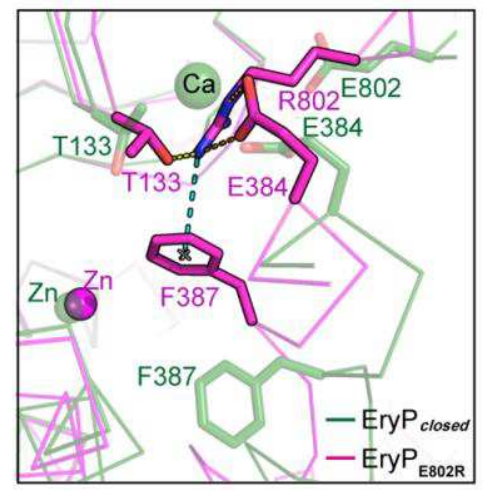

c

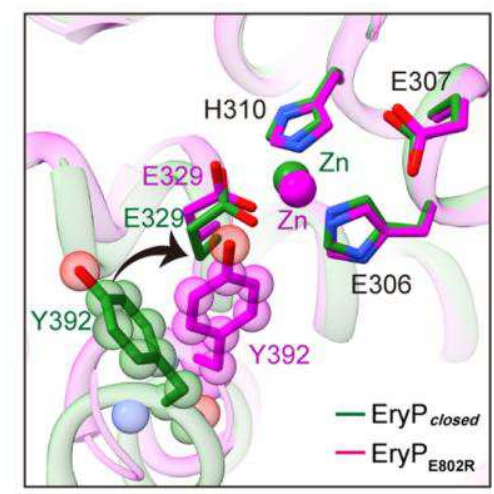

d

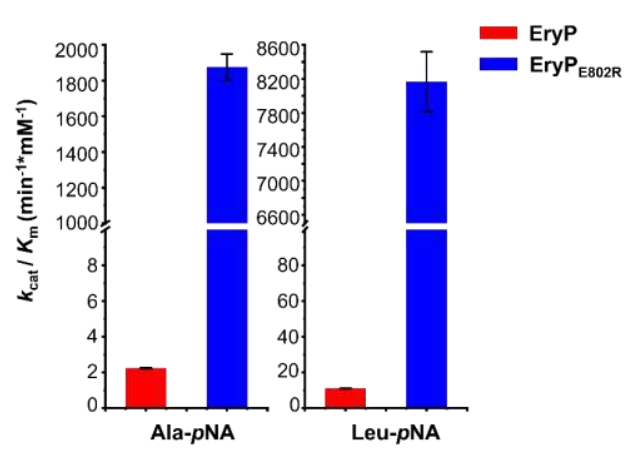

e

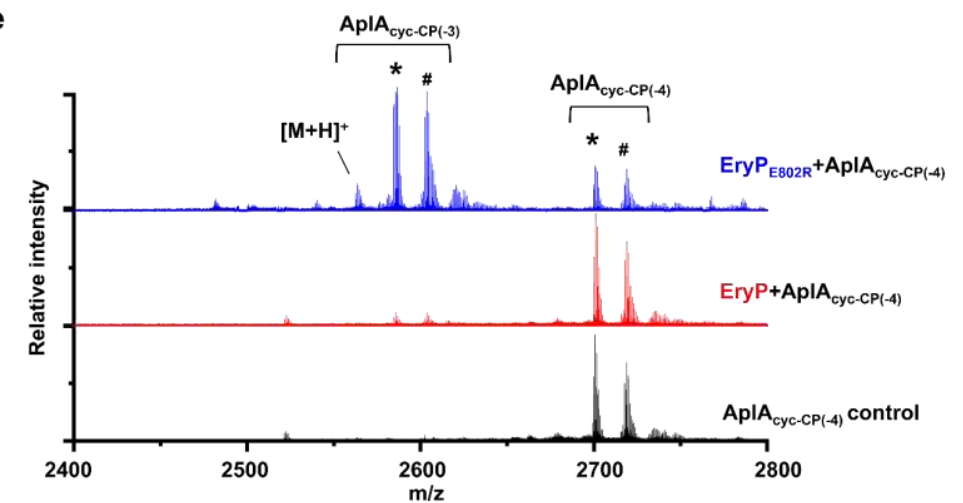

Fig. 5 | The E802R mutation enhanced the inter-domain interaction and the catalytic activity of EryP. a, Structural comparison between EryP and EryP $\mathrm{E} 802 \mathrm{R}_{\mathrm{B}} \mathbf{b}$, The remodeled inter-domain hydrogen bonding network introduced by the

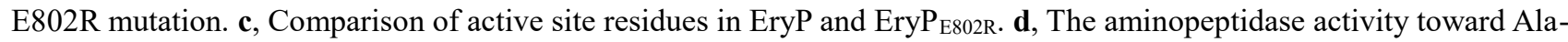
$p$ NA and Leu- $p$ NA was significantly enhanced. Error bars indicate standard deviation of three independent replicates. e, The aminopeptidase activity toward $\mathrm{AplA}_{\mathrm{cyc}-\mathrm{CP}(-4)}$ peptide was significantly enhanced. Assay conditions: $20 \mathrm{mM}$ Tris, $p \mathrm{H}$ 8.0, $100 \mu \mathrm{M}$ AplA cyc peptide, $1.0 \mu \mathrm{M}$ EryP or EryP $\mathrm{E} 802 \mathrm{R}_{\text {a }} 37{ }^{\circ} \mathrm{C}$ for $90 \mathrm{~min}$. AplA $\mathrm{A}_{\text {cyc-CP(-3): }} M_{\text {calc. }}{ }^{*}=2584.12 \mathrm{Da}$, 
$M_{\text {obs. }}{ }^{*}=2584.69 \mathrm{Da} ; \mathrm{AplA}_{\mathrm{cyc}-\mathrm{CP}(-4)}: M_{\text {calc. }}{ }^{*}=2699.15 \mathrm{Da}, M_{\mathrm{obs} .}{ }^{*}=2699.94 \mathrm{Da} .{ }^{*}$ and ${ }^{\#}$ represents the sodium and potassium

262 adducts of peptides in MS, respectively.

263 Engineering of inter-domain interactions as a general strategy to modulate the activity of LanPM1 enzymes. The

264 successful engineering of EryP by remodeling the inter-domain interaction prompted us to examine the general 265 applicability of this strategy for other LanP $\mathrm{M}_{\mathrm{M}}$ enzymes. AplP, the protease from NAI-112 biosynthesis and the first example 266 of LanP $\mathrm{M}_{\mathrm{M}}$ proteases, was selected as a model. Based on the high sequence similarities between EryP and AplP (49.5\%), 267 we built a model of AplP using EryP structure (closed) as a template to analyze the potential domain interface. Referring to the T133-E384-R802 inter-domain hydrogen bonding network in EryP $\mathrm{E}_{\mathrm{E} 82 \mathrm{R}}$, we speculated that the incorporation of two 269 Glu residues and an Arg residue at positions 98, 368 and 779 of AplP might also introduce charge-charge interactions and strengthen the inter-domain association (Supplementary Fig. 17). Thus, we prepared AplP $\mathrm{R}_{\mathrm{R} 98 \mathrm{E}-\mathrm{A} 368 \mathrm{E}-\mathrm{A} 779 \mathrm{R}}$ and evaluated its

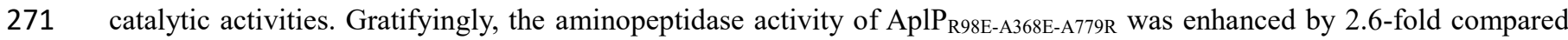
272 with AplP toward Ala- $p$ NA (Supplementary Fig. 18). Furthermore, AplP R98E-A368E-A779R displayed significantly higher 273 endopeptidase activity toward its native peptide substrate AplA $\mathrm{A}_{\mathrm{cyc}}$ than wild-type AplP, but with altered preference to 274 cleavage sites (Fig. 6a). During prolonged incubation, AplP generated AplA $\mathrm{A}_{\mathrm{cyc}-\mathrm{CP}(-4)}$ as the major product but no further 275 trimming was detected, indicating that residue Glu(-4) is a challenging residue to remove (Fig. 6b). In contrast, AplP $\mathrm{P}_{\mathrm{R} 9 \mathrm{E}-}$ A368E-A779R exhibited enhanced aminopeptidase activity by overcoming residue Glu(-4) and further produced AplA $\mathrm{A}_{\text {cyc-CP(-1) }}$ 277 as a major product. Therefore, we have constructed an AplP mutant with improved proteolytic activity for the removal of AplA leader peptide through rational introduction of bridging residues that strengthen the inter-domain interaction, which might serve as a general strategy to engineer $\mathrm{LanP}_{\mathrm{M} 1}$ proteases for improved efficiency during leader removal of corresponding lanthipeptides. 


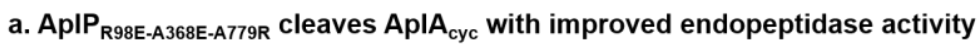

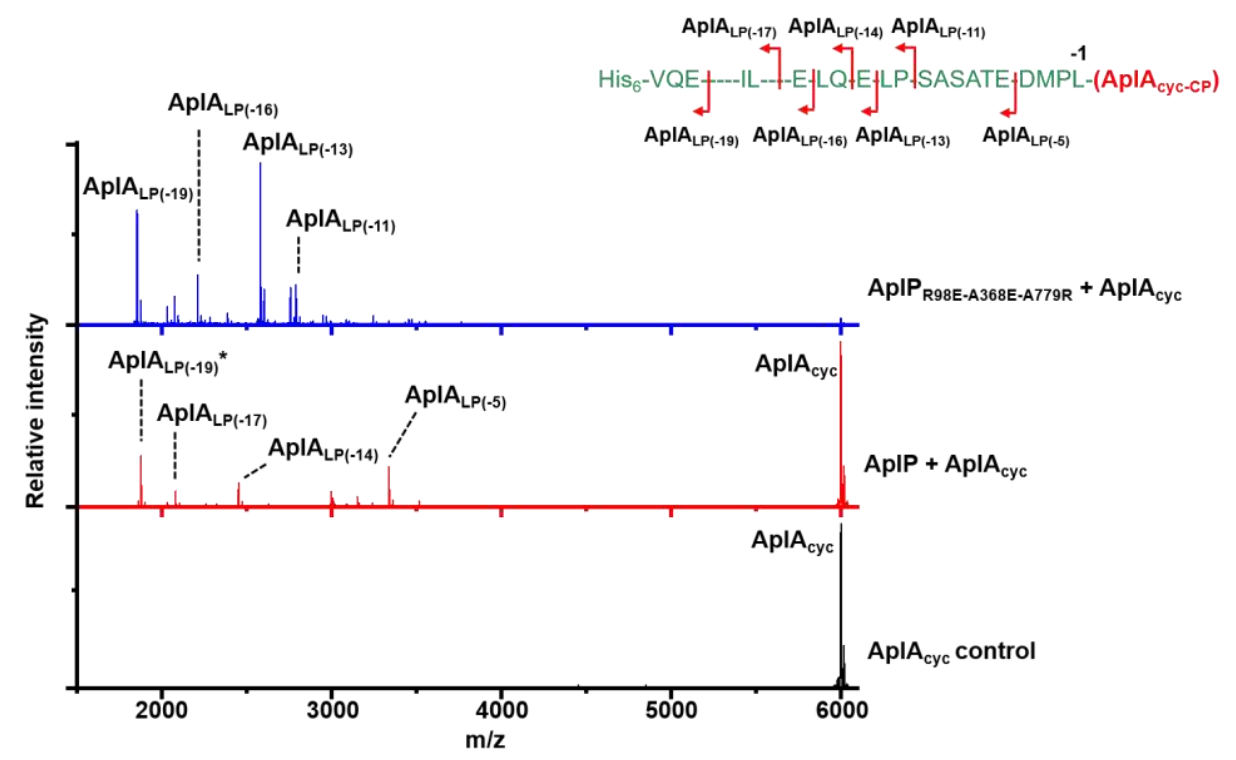

b. ApIP $P_{\mathrm{R} 98 \mathrm{E}-\mathrm{A} 368 \mathrm{E}-\mathrm{A} 779 \mathrm{R}}$ displays improved aminopeptidase activity during prolonged incubation

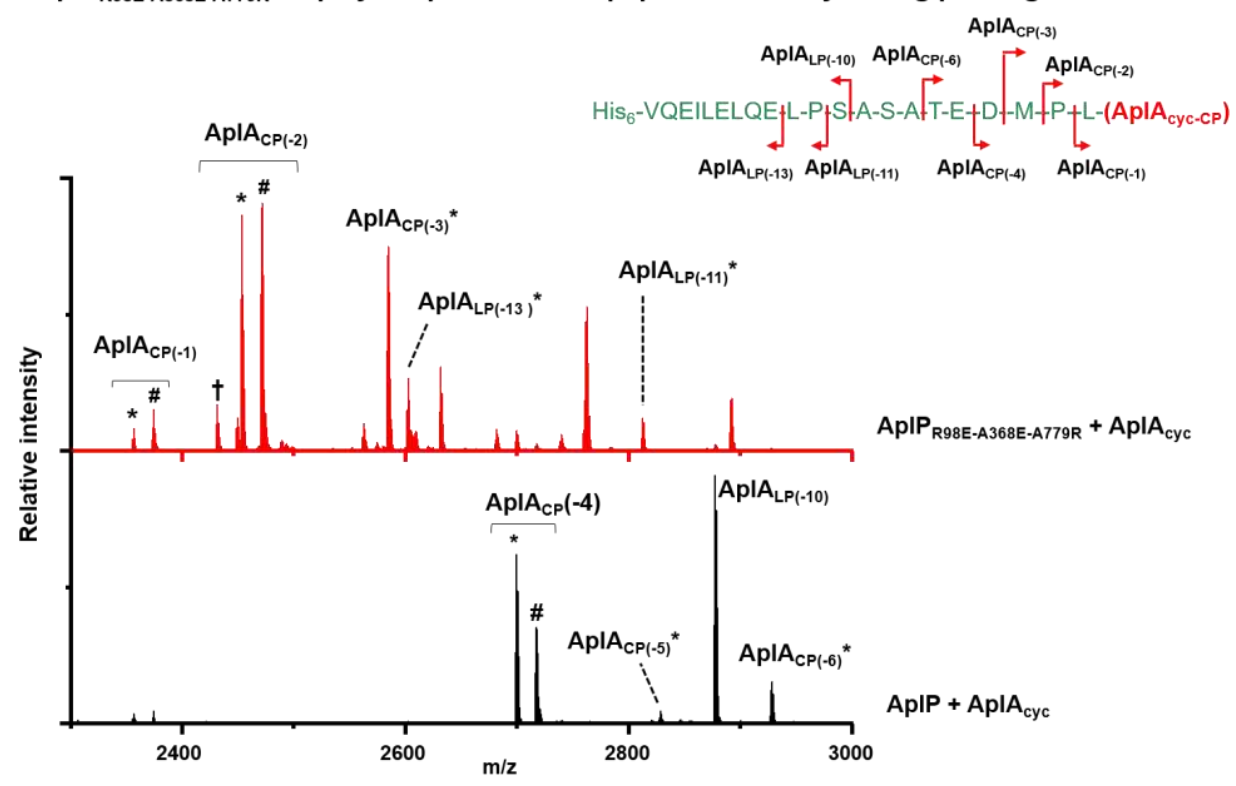

Fig. 6 | ApIPR98E-A368E-A779R display improved efficiency in removing the LP of AplAcyc peptide in comparison with

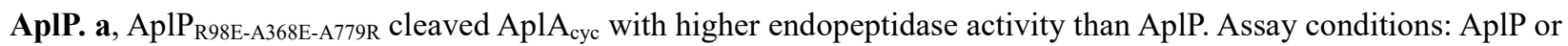
$\operatorname{AplP}_{\text {R98E-A368E-A779R }}(10 \mu \mathrm{M})$ and AplAcyc $(100 \mu \mathrm{M})$ were incubated in $20 \mathrm{mM}$ Tris buffer, $p \mathrm{H} 8.0$, at $37^{\circ} \mathrm{C}$ for $1 \mathrm{~h} . \mathbf{b}$,

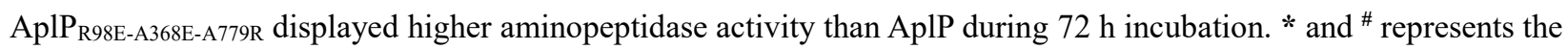
sodium and potassium adducts of peptides in MS, respectively. Detailed MS data are provided in Supplementary Table $3 \& 4$.

\section{DISCUSSION}

A molecular ruler model has been proposed to explain the substrate preference toward peptides of certain length and 
bind to a regulatory site in ERAP1 and induce allosteric activation of ERAP1 by shifting its conformation toward the closed form. ${ }^{14,24}$ In contrast, the presence of peptide substrates has little impact on the hydrolytic efficiency of EryP toward Ala-pNA under assay conditions (Supplementary Fig. 19). In addition, the inter-domain cavity in the closed form of EryP is $\sim 1530 \AA^{3}$, which is not sufficient to fully accommodate the cyclized core peptide of AplA peptide $\left(\sim 2300 \AA^{3}\right)$. These data implicate that EryP might have distinct mechanisms of substrate recognition and enzyme activation. Although EryP binds to full length Ery $A_{L P}$ and $A p l A_{L P}$ peptides (Table 1), it shows no measurable binding with endoproteolytic products $\operatorname{EryA}_{\mathrm{LP}(-15)-(-1)}$ and $\mathrm{AplA}_{\mathrm{LP}(-6)-(-1)}$ and displays no aminopeptidase activity toward them even after long time incubation (Supplementary Fig. 20). However, EryP could recognize and trim the C-terminal products with cyclized core peptide

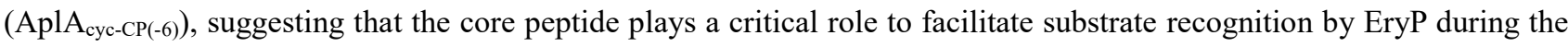
second phase of leader removal. Incubation of EryP and AplA $A_{L P(-6)}$ in trans with $A_{p l} A_{c y c-C P}$ peptide did not lead to improved

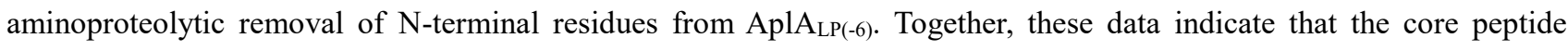
Apl $A_{\text {cyc-CP }}$ functions as a substrate recognition unit to deliver the $A p 1 A_{L P(-6)}$ segment to EryP, instead of as an allosteric activator. Therefore, we propose that the apparent low aminopeptidase activity of EryP during the second phase of leader removal is primarily due to the low binding affinity to peptide substrates.

Three sets of EryP crystal structures, the open, intermediate and closed states, demonstrate that domain-IV are highly dynamic with large-scale motions relative to domain-I and II (Fig. 3a). The dynamic domain motion is a critical evolutionary adaptation for M1 aminopeptidases to act as efficient trimming enzymes and regulates the catalytic efficiency indirectly. A unique inter-domain Ca binding site was visualized in the closed state of EryP, which bridges coordinating residues from domain-I, II and IV, thereby stabilizing the inter-domain association. Unlike thermolysin, ${ }^{31}$ the Ca binding is not strictly required for the folding or proteolytic activity of EryP, and mutations of Ca binding site have no negative impact on its activity (Supplementary Fig. 12). However, the binding of Ca ions drives the conformational equilibrium of EryP toward the close state and enhanced its aminopeptidase activity. Thus, this Ca binding site appears to be the key regulatory element with direct impact on its aminopeptidase activity. The direct correlation between the dynamic conformational changes and enzymatic activity is further demonstrated by the $\mathrm{EryP}_{\mathrm{E} 802}$ mutant with a more compact and stabilized closed structure and aminopeptidase activity enhanced by over 700 -fold compared with EryP. This result could be rationalized by considering that the long-range attractive electrostatic forces introduced by the E802R mutation facilitates the domain motion. Therefore, our observation strongly supports the proposal from previous studies that the conformational closure of M1 metallopeptidases is mandatory for optimal aminopeptidase activity, while the reciprocal open-close transition promotes the substrate binding and product release. ${ }^{14,22,26}$ Importantly, elucidation of the Ca binding site as the conformational regulatory element of $\mathrm{LanP}_{\mathrm{M} 1}$ enzymes allows their engineering for improved aminopeptidase activity. The successful construction of EryP $\mathrm{P}_{\mathrm{E} 802 \mathrm{R}}$ and AplP R98E-A368E-A779R with improved activities support our working 
principle, providing a practical engineering strategy for improved LanP $\mathrm{P}_{\mathrm{M} 1}$ enzymes for lanthipeptide production.

Metallopeptidases with endopeptidase activities, such as astacin and human matrix metalloproteinase-1, contain a catalytic groove lying between the helices bundle and Greek key-like beta sheet to accommodate peptide substrates. ${ }^{32}$ However, the N-terminal cleft of the substrate binding groove in M1 family metallopeptidases is largely blocked by domain I, although preserves space to lodge the substrate N-terminus. ${ }^{25,}{ }^{26,33}$ In comparison to other M1 metallopeptidases, EryP has a relatively elongated groove extending from the catalytic site with potential binding subsites to accommodate longer

N-terminal fragment of peptide substrates. This enlarged peptide binding site might facilitate EryP to achieve recognition of leader peptides and perform endoproteolytic cleavage. The V(L/Q)E(I/L)(Q/L)EL motif in Ery $A_{L P}$ and $A_{p l} A_{L P}$, which is highly conserved in class III lanthipeptide leader peptides for substrate recognition by lanthipeptide synthetases, appears to be important for LanP $\mathrm{M}_{\mathrm{M} 1}$ enzymes. Major endoproteolytic cleavage sites by EryP enzymes often reside in the middle or to the C-terminal to this conserved site (Fig. 2a; Supplementary Fig. 5), suggesting that this motif might associate to the substrate binding groove around the active site of EryP. Nevertheless, how LanP $\mathrm{M}_{\mathrm{M} 1}$ enzymes switch functions between endo- and aminopeptidases requires further investigation.

In conclusion, we report the biochemical and structural characterization of bifunctional M1 zinc-metallopeptidase EryP. Crystal structures of EryP in different states provide insights into the conformational dynamics of the rate-limiting protease LanP $\mathrm{P}_{\mathrm{M} 1}$ enzymes in lanthipeptide biosynthesis. An unprecedented inter-domain $\mathrm{Ca}$ binding site is revealed as an effector mechanism for inter-domain association and aminopeptidase activity. These results not only highlight the importance of the conformation dynamics in M1 metallopeptidase function but also provide valuable insights with respect to understanding their trimming mechanism. Importantly, strengthening the inter-domain interactions through rationally designing bridging residues generates $\mathrm{LanP}_{\mathrm{M} 1}$ variants with improved aminopeptidase activities, which hold potential application in the bioengineering and production of lanthipeptides. This successful engineering effort could potentially guide site-specific protein engineering on other M1 metallopeptidases to improve their catalytic efficiency.

\section{References}

1. Rawlings, N.D., Barrett, A.J. \& Bateman, A. MEROPS: the peptidase database. Nucleic Acids Res. 38, D227-233 (2010).

2. Chandu, D., Kumar, A. \& Nandi, D. PepN, the major Suc-LLVY-AMC-hydrolyzing enzyme in Escherichia coli, displays functional similarity with downstream processing enzymes in Archaea and eukarya. Implications in cytosolic protein degradation. J. Biol. Chem. 278, 5548-5556 (2003).

3. Chandu, D. \& Nandi, D. PepN is the major aminopeptidase in Escherichia coli: insights on substrate specificity and role during sodium-salicylate-induced stress. Microbiology 149, 3437-3447 (2003).

4. Tamura, N., Lottspeich, F., Baumeister, W. \& Tamura, T. The role of tricorn protease and its aminopeptidase-interacting factors in cellular protein degradation. Cell 95, 637-648 (1998). 
$3545 . \quad$ Hermans, S.J. et al. Crystal structure of human insulin-regulated aminopeptidase with specificity for cyclic peptides. Protein Sci. 24, 190-199 (2015).

6. Nguyen, T.T. et al. Structural basis for antigenic peptide precursor processing by the endoplasmic reticulum aminopeptidase ERAP1. Nat. Struct. Mol. Biol. 18, 604-613 (2011).

7. Zervoudi, E. et al. Rationally designed inhibitor targeting antigen-trimming aminopeptidases enhances antigen presentation and cytotoxic T-cell responses. Proc. Natl. Acad. Sci. U S A 110, 19890-19895 (2013).

8. Montalban-Lopez, M. et al. New developments in RiPP discovery, enzymology and engineering. Nat. Prod. Rep. 38, 130-239 (2020).

9. Repka, L.M., Chekan, J.R., Nair, S.K. \& van der Donk, W.A. Mechanistic Understanding of Lanthipeptide Biosynthetic Enzymes. Chem. Rev. 117, 5457-5520 (2017).

10. Kozakai, R. et al. Acyltransferase that catalyses the condensation of polyketide and peptide moieties of goadvionin hybrid lipopeptides. Nat. Chem. 12, 869-877 (2020).

11. Wiebach, V. et al. The anti-staphylococcal lipolanthines are ribosomally synthesized lipopeptides. Nat. Chem. Biol. 14, 652-654 (2018).

12. Hegemann, J.D. \& Süssmuth, R.D. Matters of class: coming of age of class III and IV lanthipeptides. RSC Chem. Biol. 1, 110-127 (2020).

13. Chen, S. et al. Zn-dependent bifunctional proteases are responsible for leader peptide processing of class III lanthipeptides. Proc. Natl. Acad. Sci. U S A 116, 2533-2538 (2019).

14. Nguyen, T.T. et al. Structural basis for antigenic peptide precursor processing by the endoplasmic reticulum aminopeptidase ERAP1. Nat. Struct. Mol. Biol. 18, 604-613 (2011).

15. Ren, H., Shi, C., Bothwell, I.R., van der Donk, W.A. \& Zhao, H. Discovery and Characterization of a Class IV Lanthipeptide with a Non-overlapping Ring Pattern. ACS. Chem. Biol. 15, 1642-1649 (2020).

16. Wiebach, V. et al. An Amphipathic Alpha-Helix Guides Maturation of the Ribosomally-Synthesized Lipolanthines. Angew. Chem. Int. Ed. 59, 16777-16785 (2020).

17. Lazdunski, C., Busuttil, J. \& Lazdunski, A. Purification and properties of a periplasmic aminoendopeptidase from Escherichia coli. Eur. J. Biochem. 60, 363-369 (1975).

18. Mccaman, M.T. \& Villarejo, M.R. Structural and Catalytic Properties of Peptidase-N from EscherichiaColi K-12. Arch. Biochem. Biophys. 213, 384-394 (1982).

19. Voller, G.H. et al. Characterization of new class III lantibiotics--erythreapeptin, avermipeptin and griseopeptin from Saccharopolyspora erythraea, Streptomyces avermitilis and Streptomyces griseus demonstrates stepwise N-terminal leader processing. Chembiochem 13, 1174-1183 (2012).

20. Cerda-Costa, N. \& Gomis-Ruth, F.X. Architecture and function of metallopeptidase catalytic domains. Protein Sci. 23, 123-144 (2014).

21. Thompson, M.W., Archer, E.D., Romer, C.E. \& Seipelt, R.L. A conserved tyrosine residue of Saccharomyces cerevisiae leukotriene A4 hydrolase stabilizes the transition state of the peptidase activity. Peptides 27, 1701-1709 (2006).

22. Papakyriakou, A. \& Stratikos, E. The Role of Conformational Dynamics in Antigen Trimming by Intracellular Aminopeptidases. Front. Immunol. 8, 946 (2017). 
23. Pettersen, E.F. et al. UCSF Chimera--a visualization system for exploratory research and analysis. $J$. Comput. Chem. 25, 1605-1612 (2004).

24. Kochan, G. et al. Crystal structures of the endoplasmic reticulum aminopeptidase-1 (ERAP1) reveal the molecular basis for N-terminal peptide trimming. Proc. Natl. Acad. Sci. U S A 108, 7745-7750 (2011).

25. Kyrieleis, O.J., Goettig, P., Kiefersauer, R., Huber, R. \& Brandstetter, H. Crystal structures of the tricorn interacting factor F3 from Thermoplasma acidophilum, a zinc aminopeptidase in three different conformations. J. Mol. Biol. 349, 787-800 (2005).

26. Addlagatta, A., Gay, L. \& Matthews, B.W. Structure of aminopeptidase N from Escherichia coli suggests a compartmentalized, gated active site. Proc. Natl. Acad. Sci. U S A 103, 13339-13344 (2006).

27. Tian, W., Chen, C., Lei, X., Zhao, J. \& Liang, J. CASTp 3.0: computed atlas of surface topography of proteins. Nucleic Acids Res. 46, W363-W367 (2018).

28. Cadel, S., Darmon, C., Pernier, J., Herve, G. \& Foulon, T. The M1 family of vertebrate aminopeptidases: role of evolutionarily conserved tyrosines in the enzymatic mechanism of aminopeptidase B. Biochimie 109, 67-77 (2015).

29. Tholander, F. et al. Structure-based dissection of the active site chemistry of leukotriene A4 hydrolase: implications for M1 aminopeptidases and inhibitor design. Chem. Biol. 15, 920-929 (2008).

30. Sui, L. \& Guo, H.C. ERAP1 binds peptide C-termini of different sequences and/or lengths by a common recognition mechanism. Immunobiology 226, 152112 (2021).

31. Eijsink, V.G., Matthews, B.W. \& Vriend, G. The role of calcium ions in the stability and instability of a thermolysin-like protease. Protein Sci. 20, 1346-1355 (2011).

32. Bode, W., Gomis-Ruth, F.X., Huber, R., Zwilling, R. \& Stocker, W. Structure of astacin and implications for activation of astacins and zinc-ligation of collagenases. Nature 358, 164-167 (1992).

33. Ito, K. et al. Crystal structure of aminopeptidase N (proteobacteria alanyl aminopeptidase) from Escherichia coli and conformational change of methionine 260 involved in substrate recognition. J. Biol. Chem. 281, 33664-33676 (2006).

\section{Acknowledgments}

This work is supported by NSF of China (Grant 21922703 and 91953112 to H.W.; 81871615 and 81670008 to R. B.), the Natural Science Foundation of Jiangsu Province (Grant BK20190004 and BK20200335), the Fundamental Research Funds for the Central Universities (Grant 14380138 and 14380131 to H.W.), Ministry of Science and Technology of the People's Republic of China (Grant No.2018ZX09201018-005) and National Mega-project for Innovative Drugs (2019ZX09721001-001-001). We thank National Center for Protein Sciences Shanghai (NCPSS) beamlines BL18U and BL19U allowance. We thank the staffs of National Center for Protein Sciences Shanghai (NCPSS) beamlines BL18U and BL19U and Shanghai Synchrotron Radiation Facility (SSRF) BL17U, Shanghai, People's Republic of China, for assistance during data collection. We thank the High Performance Computing Center (HPCC) of Nanjing University for the numerical calculations on its blade cluster system. 
430 H.W. and R.B. initiated and directed this study together with W.W.. W.S., C.Z. and Y.W. prepared enzymes and peptides 431 and performed biochemical assays. C.Z. performed structural biology experiments. W.W. performed computational studies 432 on protein dynamics. All authors participate in the data analysis and manuscript preparation.

\section{Competing interests}

434 The authors declare no competing interests.

\section{$435 \quad$ Additional information}

436 Supplementary information is available for this paper online.

437 Correspondence and requests for materials should be addressed to H.W. or R.B.

438 Data availability

439 All crystal structures have been deposited in the PDB database under accession number $7 \mathrm{~V} 9 \mathrm{~N}$ for EryP $\mathrm{P}_{\text {closed }}, 7 \mathrm{~V} 9 \mathrm{P}$ for $440 \quad$ EryP $_{\text {intermediate, }} 7 \mathrm{~V} 9 \mathrm{Q}$ for EryP $\mathrm{P}_{\text {open }}$ and $7 \mathrm{~V} 9 \mathrm{O}$ for EryP $\mathrm{P}_{\mathrm{E} 802 \mathrm{R}}$. 
443 General methods. Polymerase chain reactions (PCR) were carried out on a C1000 Touch ${ }^{\mathrm{TM}}$ thermal cycler (Bio-Rad). 444 DNA sequencing was performed by the Genscript Biotech, using appropriate primers. Matrix-assisted laser desorption/ionization time-of-flight mass spectrometry (MALDI-TOF MS) was carried out on Bruker UltraFlextreme. Liquid chromatography electrospray ionization tandem mass spectrometry (LC/ESI-MS/MS) was carried out and processed using a Triple TOF 4600 System (AB Sciex) equipped with a Prominence Ultra-Fast Liquid Chromatography (UFLC) system (Shimadzu). UV-Vis spectrometry was recorded by Cary 300 (Agilent Technologies). Conditions for all ESI-MS and MS/MS were set as follows: nebulizer gas: 55 psi; heater gas: 55 psi; curtain gas: 35 psi; drying temperature: $550{ }^{\circ} \mathrm{C}$; ion spray voltage: $5500 \mathrm{~V}$; declustering potential: $100 \mathrm{~V}$; collision energy: $35 \mathrm{~V}$ (positive); collision energy spread: $10 \mathrm{~V}$. The mass range and accumulation time are 400-4000 m/z, $250 \mathrm{~ms}$ for ESI-MS and 100-2000 m/z, $100 \mathrm{~ms}$ for MS/MS, respectively. Collision-induced dissociation (CID) was performed for fragmentation of the respective peptide ions. Calibration solutions purchased from AB SCIEX were used for instrument calibration, and high resolution was chosen in the ESI+ mode.

Materials. All oligonucleotides were purchased from Genscript Biotech (Nanjing, China). Restriction endonucleases and T4 DNA ligase were purchased from New England Biolabs (Ipswich, MA, USA). Phanta ${ }^{\circledR}$ Max Master Mix was purchased from Vazyme Biotech (Nanjing, China). Media components for bacterial cultures were purchased from Thermo Fisher (Waltham, MA, USA). Chemicals were purchased from Aladdin Reagent (Shanghai, China) or from Sigma-Aldrich (Schnelldorf, Germany) unless noted otherwise. Endoprotease GluC was purchased from Roche Biosciences (Basel, Switzerland). E. coli DH5 $\alpha$ was used as the host for cloning and plasmid propagation, and E. coli BL21 (DE3) was used as a host for expression of proteins and peptides.

General nomenclature. In this report, we use the standardized nomenclature recommended by the lanthipeptide community in 2020 (Nat. Prod. Rep. 2020, 38, 130-239). The numbering of residues in lanthipeptide precursor peptides starts with the first residue of the core peptide. Residues in the leader peptide are indicated with negative numbers counting back from the junction between the leader peptide and core peptide.

Molecular cloning of $a p l A$, aplKC, aplP and $\operatorname{eryP}$ genes. Plasmids containing target genes were synthesized by Genscript Biotech and PCR amplified by 30 cycles of denaturing $\left(95^{\circ} \mathrm{C}\right.$ for $\left.30 \mathrm{~s}\right)$, annealing $\left(65^{\circ} \mathrm{C}\right.$ for $\left.30 \mathrm{~s}\right)$, and extending $\left(72{ }^{\circ} \mathrm{C}\right.$, $1 \mathrm{~min} / \mathrm{kb}$ ) using high fidelity Phanta ${ }^{\circledR}$ DNA Polymerase. Amplifications were confirmed by $2 \%$ agarose gel electrophoresis, and the PCR products were purified using an Omega Biotech Cycle Pure Kit. The target DNA fragment and pRSFDuet-1 vector were digested in separate reactions containing 1X NEB buffer (New England Biolabs) with selected pair of restriction enzymes for $2 \mathrm{~h}$ at $37^{\circ} \mathrm{C}$. The digested products were purified by agarose gel electrophoresis, and the DNA fragments were extracted from the gel using an Omega Biotech Gel Extraction Kit. The resulting DNA products were 
ligated at $16{ }^{\circ} \mathrm{C}$ for $12 \mathrm{~h}$ in $1 \mathrm{X}$ T4 DNA Ligase buffer with T4 DNA Ligase $(0.7 \mathrm{U} / \mu \mathrm{L})$. E. coli DH5 $\alpha$ cells were transformed with $2.5 \mu \mathrm{L}$ of the ligation product by heat shock, and cells were plated on LB-kanamycin agar plates and grown for $15 \mathrm{~h}$ at $37^{\circ} \mathrm{C}$. Several colonies were picked and used to inoculate separate $5 \mathrm{~mL}$ cultures of LB-kanamycin medium. The cultures were grown at $37^{\circ} \mathrm{C}$ for $12 \mathrm{~h}$, and plasmids were isolated using an Omega Biotech Plasmid Mini Kit. The sequences of the resulting plasmid products were confirmed by DNA sequencing.

Mutagenesis of ApIP and EryP. Mutagenesis of AplP and EryP was carried out by a two-stage protocol, which starts with the generation of primers using two parallel asymmetric PCRs, followed by an exponential whole-plasmid amplification. PCR amplification was performed by 30 cycles of denaturing $\left(95^{\circ} \mathrm{C}\right.$ for $\left.30 \mathrm{~s}\right)$, annealing $\left(65^{\circ} \mathrm{C}\right.$ for $\left.30 \mathrm{~s}\right)$, and extending $\left(72{ }^{\circ} \mathrm{C}, 1 \mathrm{~min} / \mathrm{kb}\right)$ using high fidelity Phanta ${ }^{\circledR}$ DNA Polymerase and confirmed by $1 \%$ agarose gel electrophoresis. PCR products were purified using an Omega Biotech Cycle Pure Kit. The target DNA fragment was digested by DpnI in NEB buffer (New England Biolabs) for $3 \mathrm{~h}$ at $37^{\circ} \mathrm{C}$. E. coli DH5 $\alpha$ cells were transformed with $2.5 \mu \mathrm{L}$ of the digested product by heat shock, plated on LB-agar plates and grown for $15 \mathrm{~h}$ at $37^{\circ} \mathrm{C}$. Single colonies were picked to inoculate separate 5 $\mathrm{mL}$ cultures of LB-chloramphenicol medium, which were grown at $37^{\circ} \mathrm{C}$ for $12 \mathrm{~h}$. Plasmids were isolated using an Omega Biotech Plasmid Mini Kit. The sequences of the resulting plasmid products were confirmed by DNA sequencing.

Overexpression and purification of AplAcyc peptide. E. coli BL21(DE3) cells were transformed with pRSFDuet-1-His6AplA and pACYCDuet-1- His $_{6}-\mathrm{AplKC}$ and plated on a Luria broth (LB) agar plate containing $50 \mathrm{mg} / \mathrm{L}$ of kanamycin and $35 \mathrm{mg} / \mathrm{L}$ of chloramphenicol. A single colony was used to inoculate a $5 \mathrm{~mL}$ culture of $\mathrm{LB}$ supplemented with $50 \mathrm{mg} / \mathrm{L}$ of kanamycin and $35 \mathrm{mg} / \mathrm{L}$ of chloramphenicol at $37^{\circ} \mathrm{C}$ for $12 \mathrm{~h}$. The culture was used to inoculate $4 \mathrm{~L}$ of LB containing 50 $\mathrm{mg} / \mathrm{L}$ of kanamycin and $35 \mathrm{mg} / \mathrm{L}$ of chloramphenicol. Cells were grown at $37^{\circ} \mathrm{C}$ to $\mathrm{OD}_{600} \sim 0.6-0.8$, cooled to $16^{\circ} \mathrm{C}$ before IPTG was added to a final concentration of $0.2 \mathrm{mM}$. The resulting cultures were grown for another $20 \mathrm{~h}$, harvested and processed following the purification procedure described in the previous section.

Overexpression and purification of EryP and ApIP enzymes. E. coli BL21 (DE3) cells were transformed with pET-28a plasmids containing genes encoding target enzymes. A single colony transformant was used to inoculate a $30 \mathrm{~mL}$ culture of LB supplemented with $50 \mu \mathrm{g} / \mathrm{mL}$ kanamycin. The culture was grown at $37^{\circ} \mathrm{C}$ for $12 \mathrm{~h}$ and was used to inoculate $3 \mathrm{~L}$ of LB containing $50 \mu \mathrm{g} / \mathrm{mL}$ kanamycin, and cells were grown at $37^{\circ} \mathrm{C}$ to $\mathrm{OD}_{600} \approx 0.6$. The culture was incubated at $4{ }^{\circ} \mathrm{C}$ on ice for $20 \mathrm{~min}$, then IPTG was added to a final concentration of $0.2 \mathrm{mM}$ and the culture was incubated at $18{ }^{\circ} \mathrm{C}$ for $20 \mathrm{~h}$. Cells were harvested by centrifugation at $12,000 \times \mathrm{g}$ for $15 \mathrm{~min}$ at $4{ }^{\circ} \mathrm{C}$, and the pellet was resuspended in $30 \mathrm{~mL}$ of start buffer (20 mM Tris buffer, $p \mathrm{H} 8.0,500 \mathrm{mM} \mathrm{NaCl}, 1.0 \mathrm{mM}$ TCEP, $10 \%$ glycerol) and stored at $-80{ }^{\circ} \mathrm{C}$. All protein purification steps were performed at $4{ }^{\circ} \mathrm{C}$. The cell paste was suspended in start buffer and the cells were lysed using a high pressure homogenizer (Avestin, Inc.). Cell debris was pelleted via centrifugation at $23,700 \times \mathrm{g}$ for $20 \mathrm{~min}$ at $4{ }^{\circ} \mathrm{C}$. The supernatant was loaded onto a $5 \mathrm{~mL}$ HisTrap HP IMAC column charged with $\mathrm{Ni}^{2+}$ and equilibrated with start buffer. The 
column was washed with $50 \mathrm{~mL}$ of buffer $\mathrm{A}(30 \mathrm{mM}$ imidazole, $20 \mathrm{mM}$ Tris, $p \mathrm{H} 7.5,300 \mathrm{mM} \mathrm{NaCl})$, and the protein was eluted using a linear gradient of $0-100 \%$ buffer B (200 mM imidazole, $20 \mathrm{mM}$ Tris, $p \mathrm{H} \mathrm{7.5,} 300 \mathrm{mM} \mathrm{NaCl})$ over $40 \mathrm{~min}$ at a $2 \mathrm{~mL} / \mathrm{min}$ flow rate. UV absorbance $(280 \mathrm{~nm})$ was monitored and fractions were collected and analyzed by SDSPAGE. The fractions containing target proteins were combined and concentrated using an Amicon Ultra-50 Centrifugal Filter Unit (30 kDa MWCO, Millipore). Gel filtration purification was used to further purify target proteins. The concentrated protein sample was injected onto an FPLC system (ÄKTA) equipped with an XK16 16/60 (GE Healthcare Life Sciences) column packed with SuperDex 75 resin previously equilibrated in $20 \mathrm{mM}$ HEPES (pH 7.5), $200 \mathrm{mM} \mathrm{KCl.}$ The protein was eluted with a flow rate of $1.0 \mathrm{~mL} / \mathrm{min}$. Both UV $(280 \mathrm{~nm})$ and conductance were monitored and fractions were collected. Misfolded/aggregated protein was efficiently separated from soluble, correctly folded protein and the desired fractions were combined and concentrated using an Amicon Ultra-15 Centrifugal Filter Unit. The resulting protein sample was stored at $-80^{\circ} \mathrm{C}$. Protein concentration was determined using a Bradford Assay Kit (Pierce).

Preparation of AplA $\mathbf{A}_{\text {cyc-CP(-4) }}$ peptide. $\mathrm{AplA}_{\mathrm{cyc}}(100 \mu \mathrm{M})$ was digested by GluC $(1.0 \mu \mathrm{M})$ in $50 \mathrm{mM}$ Tris buffer, $p \mathrm{H} 8.0$, at $37^{\circ} \mathrm{C}$ for $12 \mathrm{~h}$. The resulting AplA $\mathrm{Ayc}_{\text {cP(-4) }}$ peptide was purified by HPLC.

In vitro assay of EryP and EryP mutants cleaving EryAlP, AplAcyc and AplAcyc-CP(-4) peptides. Typically, EryP or a EryP mutant $(10 \mu \mathrm{M})$ was incubated with a peptide substrate $(100 \mu \mathrm{M})$ in $20 \mathrm{mM}$ Tris buffer, $p \mathrm{H} 8.0$, in a $37{ }^{\circ} \mathrm{C}$ water bath. EryP enzymes were omitted in negative control experiments.

In vitro assay of EryP cleaving EryA $A_{L P}$, AplA $A_{c y c}$ and $\mathbf{A p l} \mathbf{A}_{c y c-C P(-4)}$ in the presence of $\mathrm{CaCl}_{2}$. Typically, EryP or a EryP mutant $(10 \mu \mathrm{M})$ was incubated with EryA $\mathrm{LP}_{\mathrm{LP}}$ peptide $(100 \mu \mathrm{M})$ in $20 \mathrm{mM}$ Tris buffer, $p \mathrm{H} 8.0$, with or without $\mathrm{CaCl}_{2}(0.1$ $\mathrm{mM}$ or $1.0 \mathrm{mM}$ ) in a $37^{\circ} \mathrm{C}$ water bath. EryP enzymes were omitted in negative control experiments.

Reaction kinetics of the hydrolysis of amino acid-pNA derivatives by EryP and AplP. Typically, AplP, EryP (1.0 $\mu \mathrm{M})$, inactive EryP mutants $(1.0 \mu \mathrm{M})$ or $\operatorname{EryP}_{\mathrm{E} 802 \mathrm{R}}(0.02 \mu \mathrm{M})$ was incubated with amino acid- $p$ NA derivatives of varied

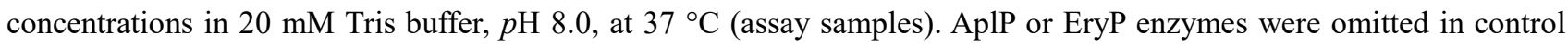
samples. The formation of product $p$-nitroanilide was measured by the absorbance at $405 \mathrm{~nm}\left(\mathrm{Abs}_{p \mathrm{NA}}=\mathrm{AbS}_{405 \mathrm{~nm} \_ \text {assay_sample}}{ }^{-}\right.$

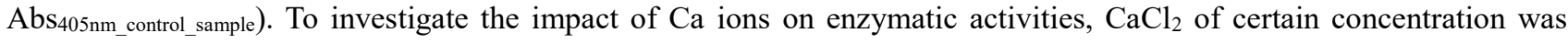
added in the assay and control buffers.

Fluorescence-labeling of peptides. FITC-labeling of $\mathrm{AplA}_{c y c}$ peptide was performed in conditions: $90 \mathrm{mM}$ phosphate buffer, $p \mathrm{H} 8.4,45 \mu \mathrm{M}$ peptide and $450 \mu \mathrm{M}$ FITC for $12 \mathrm{~h}$ at room temperature and in dark conditions. Fluorescencelabeling of Ery $A_{L P}$ and Apl $A_{L P}$ peptides was performed in conditions: $20 \mathrm{mM}$ phosphate buffer $\left(\mathrm{EtOH}: \mathrm{H}_{2} \mathrm{O}=2: 8, \mathrm{v} / \mathrm{v}\right), p \mathrm{H}$ 7.4, $10 \mu \mathrm{M}$ peptide, $20 \mu \mathrm{M}$ fluorescein o-acrylate. The reaction was allowed to proceed for $1 \mathrm{~h}$ at room temperature in dark conditions. The desired FITC-peptide conjugates were purified by HPLC.

Measurement of protein-peptide interactions by MST. As a representative example, the binding affinity between EryP 
and FITC-labeled EryA $A_{L P}$ was measured using Monolith NT.115 Pico (Nanotemper Technologies). The EryA $A_{L P}$ sample was diluted using MST buffer (50 mM Tris, $150 \mathrm{mM} \mathrm{NaCl,} \mathrm{0.05 \%} \mathrm{Tween-20,} 10 \mathrm{mM} \mathrm{MgCl} 2,5 \%$ glycerol) until pretest fluorescence excitation range from $200-2000$ for the most optimized response $(\sim 1.0 \mu \mathrm{M})$. To perform the MST assay, FITC-labeled EryA $A_{\mathrm{LP}}$ sample $(10 \mu \mathrm{L})$ was first incubated with EryP $(10 \mu \mathrm{L})$ of 16 different serial dilutions in the assay buffer for 5 min to allow binding. Samples were then loaded into Monolith NT.115 capillary (Nanotemper Technologies) and measured using 20\% (Auto-detect) Nano - BLUE as excitation power and medium MST power. The measurement was repeated for three times. Data analysis was performed using Nanotemper affinity analysis software.

Crystallization, data collection of EryP. The purified protein was adjusted to about $9 \mathrm{mg} / \mathrm{ml}$ and subjected to screenings of different crystallization conditions at $18{ }^{\circ} \mathrm{C}$. For EryP in the closed state, crystals in the space group $P 4_{3} 2{ }_{1} 2$ were grown from hanging drops with $3 \mu \mathrm{L}$ of reservoir solution $(0.1 \mathrm{M}$ calcium acetate; $0.1 \mathrm{M}$ sodium acetate, $\mathrm{pH} 4.5 ; 10 \% \mathrm{w} / \mathrm{v}$ PEG 4000). The condition for the growth of Se-Met-labeled crystals is the same as that for the native crystals in the closed state. When co-crystallized with $2 \mathrm{mM}$ VLELQE peptide, which is conserved in the leader peptides of class III lanthipeptides, crystals of EryP in the intermediate state that belongs to the space group $P 12_{1} 1$ appeared after two months under the condition of $0.2 \mathrm{M} \mathrm{NaCl}, 0.1 \mathrm{M}$ Tris, $\mathrm{pH} 8.0$ and 20\% w/v PEG 4000. When co-crystallized with 4mM cyclized-AplA, which contains the leader peptide and the cyclized core peptide, the open form crystals in the space group $P 2{ }_{1}{ }_{1} 2{ }_{1}$ were obtained by mixing $1.5 \mu \mathrm{L}$ of sample solution and $1 \mu \mathrm{L}$ of reservoir solution $(0.1 \mathrm{M}$ citric acid, $\mathrm{pH} 3.5 ; 25 \% \mathrm{w} / \mathrm{v}$ PEG3350) using the sitting method. The crystals of EryP $\mathrm{P}_{\mathrm{E} 802 \mathrm{R}}$ were grown in $0.2 \mathrm{M}$ ammonium chloride, $20 \% \mathrm{w} / \mathrm{v} \mathrm{PEG} 6000$ and MES, pH 6.0. Prior to data collection, all the crystals were transferred to cryoprotectant solutions $(20 \% \mathrm{v} / \mathrm{v}$ glycerol in corresponding crystallization solution) and flash-cooled in liquid nitrogen. X-ray data were collected at BL-17U1 or BL18U1 station of the Shanghai Synchrotron Radiation Facility (SSRF), China.

Data processing and structure determination of EryP. Diffraction images were integrated and scaled with the HKL2000 program package ${ }^{34}$. The phase problem of Se-Met-labeled EryP was solved by using the single-wavelength anomalous dispersion (SAD) method, and nine Se sites were found by using the AutoSol tool of PHENIX suite ${ }^{35}$. The native structures of EryP in different conformations were determined by molecular replacement using the Phaser-MR tool of PHENIX and the structure of Se-Met-labeled EryP was selected as a template. The structures were rebuilt with COOT $^{36}$ and refined using the Refinement tool of PHENIX or $\mathrm{CCP} 4^{37}$ for several rounds. Data collection and refinement statistics are shown in Table S1. The structural figures were drawn with PyMOL (https://pymol.org/2/) and USCF Chimera $\mathrm{X}^{38}$.

Initial structural preparation for computational studies. The initial structure of EryP with $\mathrm{Ca}^{2+}$ ion bound was based on the closed structure of EryP (PDB ID: 7V9N). The Ca ion was manually removed from the EryP closed $_{\text {structure to build }}$ an EryP structure without $\mathrm{Ca}^{2+}$ bound. The starting coordinate of the EryP $\mathrm{E}_{\mathrm{E} 802 \mathrm{R}}$ mutant were based on its crystal structure (PDB ID: 7V9O). The protonation states of charged residues were determined at constant $p \mathrm{H} 7.5$ based on $p \mathrm{~K}_{\mathrm{a}}$ 
calculations via the PROPKA program ${ }^{39}$ and the consideration of the local hydrogen bonding network. In models of EryP with/without $\mathrm{Ca}$ ion bound, residues His102, 182, 231, 249, 422, 489, 492, 512, 532, 659, 756, 774 and 797 were assigned as HIE; residues His306, 310 and 710 were set as HID; the rest His residues were HIP. In the model of EryP ${ }_{\mathrm{E} 802 \mathrm{R}}$, residues His102, 182, 306, 310, 512, 756, 710, 756 and 797 were assigned as HIE; residues His115, 231, 249, 422, 489, 492, 532, 659 and 774 were set as HID; the rest His residues were HIP. In above three models, all Asp and Glu residues were set as deprotonated status except for Asp315, while Lys and Arg resides were all set as protonated status. The force field parameters for the $\mathrm{Zn}$ center active site were prepared using the Metal Center Parameter Builder (MCPB.py) ${ }^{40}$ as implemented in Amber $16^{41}$, in which residues H306, H310, E329 and a catalytic water molecule were all treated in coordinate bonded way. Bond and angle force constants were derived using the Seminario method $^{42}$, and point charge parameters for the electrostatic potentials were obtained using the ChgModB method. Each model was neutralized by adding $\mathrm{Na}^{+}$ions and solvated into a truncated octahedron TIP $3 \mathrm{P}^{43}$ water box with a $10 \AA$ buffer distance on each side. These three models consisted of 84549,84547 and 88052 atoms for the EryP bound with or without $\mathrm{Ca}^{2+}$ and EryP $\mathrm{E}_{\mathrm{E} 02 \mathrm{R}}$, respectively.

Molecular docking. The GLIDE program ${ }^{44}$ was used to perform molecular docking of EryP with the LE peptide. The crystal structure of EryP in the closed state was minimized by using the OPLS3 force field and the ligand was prepared with the 'LigPrep' module. All docking procedures were calculated in the "Standard Precision" (SP) mode with default parameters. The final scoring was performed on energy-minimized poses and displayed as Glide scores. The best-docked pose with the lowest Glide score value was selected for further analysis.

To dock Leu- $p$ NA into the active sites of EryP enzymes, 50000 snapshots uniformly distributed at equal intervals from the last $100 \mathrm{~ns}$ MD simulation (with time intervals of $2 \mathrm{ps)} \mathrm{were} \mathrm{picked} \mathrm{up} \mathrm{and} \mathrm{divided} \mathrm{into} \mathrm{ten} \mathrm{groups} \mathrm{using}$ hierarchical agglomerative (bottom-up) approach ${ }^{45}$. Leu- $p \mathrm{NA}$, the favorite aminopeptidase substrate of EryP, was fully optimized at the B3LYP-D3/6-31+G(d) level of Gaussian 16 using the $\mathrm{CPCM}^{46-48}$ model in water, and then docked into the active site of one representative group snapshot to mimic the native substrate-EryP complex. Molecular docking was performed using the Lamarckian genetic algorithm local search method in the AutoDock 4.2 and AutoDockTools-1.5.6 ${ }^{49}$. The docking approach was employed on rigid-receptor conformation, while all the rotatable torsional bonds of Leu- $p$ NA were set free. A grid box was centered on the $\mathrm{Zn}$ atom and its size was set to $40 \AA \times 40 \AA \times 40 \AA$ points with a $0.375 \AA$ spacing. A total of 500 independent docking runs were undertaken with a maximum energy evaluation of $2.5 \times 10^{7}$. The obtained 500 docked conformations were clustered with $2.0 \AA$ RMSD and ranked depending on an energy-based scoring function. The possible catalytically active binding modes were 
selected as initial configurations to perform MD simulations of EryP and EryP $\mathrm{P}_{\mathrm{E} 802 \mathrm{R}}$ in complex with Leu- $p \mathrm{NA}$ according to scoring function and reasonable conformation.

Molecular dynamics simulation. All molecular dynamics (MD) simulations were performed by Amber 16 package $^{41}$. The MD pre-equilibrated EryP and EryP $\mathrm{E}_{\mathrm{E} 02 \mathrm{R}}$ structures and possible catalytic active binding modes of Leu- $p$ NA were used as the starting conformations for MD simulations on the protein-ligand complexes. The partial charge of Leu- $p$ NA was fitted with HF/6-31G(d) calculations and the restrained electrostatic potential (RESP) $)^{50,51}$ protocol implemented by the Antechamber module in Amber 16 package ${ }^{41}$. The force field parameters for Leu- $p$ NA were adapted from the standard general amber force field 2.0 (gaff 2$)^{52}$ parameters, while the standard Amber14SB force field was applied to describe the protein. Here, the force field parameters for the $\mathrm{Zn}$ center active site were treated in a hybrid bonded/restrained nonbonded mode in which H306, H310 and E329 were treated in bonded way while a catalytic water molecule was in nonbonded way using MCPB.py ${ }^{40}$ module. Each system was initially neutralized with $\mathrm{Na}^{+}$counter ions and solvated with explicit TIP3P ${ }^{43}$ water in a truncated octahedron box with a 10 $\AA$ buffer distance. The resulting system contained 90788 (EryP bound with $\mathrm{Ca}^{2+}$ and Leu- $p \mathrm{NA}$ ), 89937 (EryP bound with Leu- $p \mathrm{NA}$ but no $\mathrm{Ca}^{2+}$ ) and $87661\left(\mathrm{EryP}_{\mathrm{E} 802 \mathrm{R}}\right.$ bound with Leu- $p \mathrm{NA}$ but no $\mathrm{Ca}^{2+}$ ) atoms, respectively.

Each system was equilibrated with a series of minimizations interspersed by short MD simulations during which restraints on the protein backbone heavy atoms were gradually released (with force constant of 10, 2, 0.1 and 0 $\left.\mathrm{kcal} /\left(\mathrm{mol} \cdot \AA^{2}\right)\right)$, and then heated slowly from 0 to $300 \mathrm{~K}$ for $50 \mathrm{ps}$. Finally, the standard unrestrained $200 \mathrm{~ns} \mathrm{MD}$ simulation with periodic boundary condition at $300 \mathrm{~K}$ and $1 \mathrm{~atm}$ was carried out. The pressure was maintained at 1 atm and coupled with isotropic position scaling. The temperature was controlled at $300 \mathrm{~K}$ with Berendsen thermostat method. Long-range electrostatic interactions were treated with particle mesh Ewald (PME) ${ }^{53}$ method and $12 \AA$ cutoff was applied to both PME and van der Waals (vdW) interactions. Time step of 2 fs was employed along with SHAKE algorithm for hydrogen atoms, and periodic boundary condition was used. Each system was checked for stability (structure, energy, and temperature fluctuations) and convergence (root mean square deviations-RMSD of structures).

\section{References}

34. Otwinowski, Z. \& Minor, W. Processing of X-ray diffraction data collected in oscillation mode. Methods Enzymol. 276, 307-326 (1997).

35. Adams, P.D. et al. PHENIX: a comprehensive Python-based system for macromolecular structure solution. Acta Crystallogr. D Biol. Crystallogr. 66, 213-221 (2010). 
36. Emsley, P., Lohkamp, B., Scott, W.G. \& Cowtan, K. Features and development of Coot. Acta Crystallogr. 66, 486-501 (2010).

37. Winn, M.D. et al. Overview of the CCP4 suite and current developments. Acta Crystallogr. D Biol. Crystallogr. 67, 235-242 (2011).

38. Pettersen, E.F. et al. UCSF ChimeraX: Structure visualization for researchers, educators, and developers. Protein science : a publication of the Protein Society 30, 70-82 (2021).

39. Rostkowski, M., Olsson, M.H.M., Sondergaard, C.R. \& Jensen, J.H. Graphical analysis of pH-dependent properties of proteins predicted using PROPKA. BMC Structural Biol. 11, 6-12 (2011).

40. Li, P. \& Merz, K.M., Jr. MCPB.py: A python based metal center parameter builder. J. Chem. Inf. Model. 56, 599-604 (2016).

41. Case, D.A. et al. (University of California, San Francisco; 2016).

42. Seminario, J.M. Calculation of intramolecular force fields from second-derivative tensors. Int. J. Quant. Chem. 60, 1271-1277 (1996).

43. Jorgensen, W.L., Chandrasekhar, J., Madura, J.D., Impey, R.W. \& Klein, M.L. Comparison of simple potential functions for simulating liquid water. J. Chem. Phys. 79, 926-935 (1983).

44. Friesner, R.A. et al. Extra precision glide: docking and scoring incorporating a model of hydrophobic enclosure for protein-ligand complexes. J. Med. Chem. 49, 6177-6196 (2006).

45. Ward, J.H. Hierarchical Grouping to Optimize an Objective Function. J. Am. Stat. Assoc. 58, $236-244$ (1963).

46. Barone, V. \& Cossi, M. Quantum calculation of molecular energies and energy gradients in solution by a conductor solvent model. J. Phys. Chem. A 102, 1995-2001 (1998).

47. Takano, Y. \& Houk, K.N. Benchmarking the conductor-like polarizable continuum model (CPCM) for aqueous solvation free energies of neutral and ionic organic molecules. J. Chem. Theory Comput. 1, $70-77$ (2005).

48. Cossi, M., Rega, N., Scalmani, G. \& Barone, V. Energies, structures, and electronic properties of molecules in solution with the C-PCM solvation model. J. Comput. Chem. 24, 669-681 (2003).

49. Morris, G.M. et al. Automated docking using a Lamarckian genetic algorithm and an empirical binding free energy function. J. Comput. Chem. 19, 1639-1662 (1998).

50. Jakalian, A., Bush, B.L., Jack, D.B. \& Bayly, C.I. Fast, efficient generation of high-quality atomic Charges. AM1-BCC model: I. Method. J. Comput. Chem. 21, 132-146 (2000).

51. Jakalian, A., Jack, D.B. \& Bayly, C.I. Fast, efficient generation of high-quality atomic charges. AM1-BCC model: II. Parameterization and validation. J. Comput. Chem. 23, 1623-1641 (2002). 
653 52. Wang, J.M., Wolf, R.M., Caldwell, J.W., Kollman, P.A. \& Case, D.A. Development and testing of a general 654 amber force field. J. Comput. Chem. 25, 1157-1174 (2004).

655 53. Darden, T.; York, D.; Pedersen, L., Particle mesh Ewald - an n. $\log (\mathrm{n})$ method for Ewald sums in large systems. 656 J. Chem. Phys. 1993, 98 (12), 10089-10092. 


\section{Supplementary Files}

This is a list of supplementary files associated with this preprint. Click to download.

- SupplementrayfileNCB.pdf

- SupplementrayfileNCB.pdf

- Wangreportingsummary.pdf

- Wangeditorialpolicychecklist.pdf

- Validationreport7V90.pdf

- Validationreport7V9Q.pdf

- Validationreport7V9P.pdf

- Validationreport7V9N.pdf 